\title{
A "Strange" Functional Equation for Eisenstein Series and Miraculous Duality on the Moduli Stack of Bundles
}

\section{Citation}

Gaitsgory, Dennis. 2017. A “Strange" Functional Equation for Eisenstein Series and Miraculous Duality on the Moduli Stack of Bundles. Annales Scientifiques de l'École Normale Supérieure 50, no. 5: 1123-1162.

\section{Permanent link}

http://nrs.harvard.edu/urn-3:HUL.InstRepos:41363978

\section{Terms of Use}

This article was downloaded from Harvard University's DASH repository, and is made available under the terms and conditions applicable to Other Posted Material, as set forth at http:// nrs.harvard.edu/urn-3:HUL.InstRepos:dash.current.terms-of-use\#LAA

\section{Share Your Story}

The Harvard community has made this article openly available. Please share how this access benefits you. Submit a story. 


\title{
A "STRANGE" FUNCTIONAL EQUATION FOR EISENSTEIN SERIES AND MIRACULOUS DUALITY ON THE MODULI STACK OF BUNDLES
}

\author{
D. GAITSGORY
}

\begin{abstract}
We show that the failure of the usual Verdier duality on $\operatorname{Bun}_{G}$ leads to a new duality functor on the category of D-modules, and we study its relation to the operation of Eisenstein series.
\end{abstract}

\section{INTRODUCTION}

\subsection{Context for the present work.}

0.1.1. This paper arose in the process of developing what V. Drinfeld calls the geometric theory of automorphic functions. I.e., we study sheaves on the moduli stack $\operatorname{Bun}_{G}$ of principal $G$ bundles on a curve $X$. Here and elsewhere in the paper, we fix an algebraically closed ground field $k$, and we let $G$ be a reductive group and $X$ a smooth and complete curve over $k$.

In the bulk of the paper we will take $k$ to be of characteristic 0 , and by a "sheaf" we will understand an object of the derived category of D-modules. However, with appropriate modifications, our results apply also to $\ell$-adic sheaves, or any other reasonable sheaf-theoretic situation.

Much of the motivation for the study of sheaves on $\mathrm{Bun}_{G}$ comes from the so-called geometric Langlands program. In line with this, the main results of this paper have a transparent meaning in terms of this program, see Sect. 0.2. However, one can also view them from the perspective of the classical theory of automorphic functions (rather, we will see phenomena that so far have not been studied classically).

0.1.2. Constant term and Eisenstein series functors. To explain what is done in this paper we will first recall the main result of [DrGa3].

Let $P \subset G$ be a parabolic subgroup with Levi quotient $M$. The diagram of groups

$$
G \hookleftarrow P \rightarrow M
$$

gives rise to a diagram of stacks

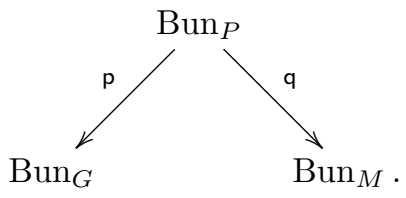

Using this diagram as "pull-push", one can write down several functors connecting the categories of D-modules on $\mathrm{Bun}_{G}$ and $\mathrm{Bun}_{M}$, respectively. By analogy with the classical theory of automorphic functions, we call the functors going from $\operatorname{Bun}_{M}$ to Bun $_{G}$ "Eisenstein series", and the functors going from $\operatorname{Bun}_{G}$ to $\operatorname{Bun}_{M}$ "constant term".

Date: May 9, 2016. 
Namely, we have

$$
\begin{array}{ll}
\text { Eis! }:=\mathrm{p}_{!} \circ \mathrm{q}^{*}, & \mathrm{D}-\bmod \left(\operatorname{Bun}_{M}\right) \rightarrow \mathrm{D}-\bmod \left(\operatorname{Bun}_{G}\right), \\
\mathrm{Eis}_{*}:=\mathrm{p}_{*} \circ \mathrm{q}^{!}, & \mathrm{D}-\bmod \left(\operatorname{Bun}_{M}\right) \rightarrow \mathrm{D}-\bmod \left(\operatorname{Bun}_{G}\right), \\
\mathrm{CT}_{!}:=\mathrm{q}_{!} \circ \mathrm{p}^{*}, & \mathrm{D}-\bmod \left(\operatorname{Bun}_{G}\right) \rightarrow \mathrm{D}-\bmod \left(\operatorname{Bun}_{M}\right), \\
\mathrm{CT}_{*}:=\mathrm{q}_{*} \circ \mathrm{p}^{!}, & \mathrm{D}-\bmod \left(\operatorname{Bun}_{G}\right) \rightarrow \mathrm{D}-\bmod \left(\operatorname{Bun}_{M}\right) .
\end{array}
$$

Note that unlike the classical theory, where there is only one pull-back and one push-forward for functions, for sheaves there are two options: ! and $*$, for both pull-back and push-forward. The interaction of these two options is one way to look at what this paper is about.

Among the above functors, there are some obvious adjoint pairs: Eis! is the left adjoint of $\mathrm{CT}_{*}$, and $\mathrm{CT}_{\text {! }}$ is the left adjoint of $\mathrm{Eis}_{*}$.

In addition to this, the following, perhaps a little unexpected, result was proved in [DrGa3]:

Theorem 0.1.3. The functors $\mathrm{CT}_{!}$and $\mathrm{CT}_{*}^{-}$are canonically isomorphic.

In the statement of the theorem the superscript "_" means the constant term functor taken with respect to the opposite parabolic $P^{-}$(note that the Levi quotients of $P$ and $P^{-}$are canonically identified).

Our goal in the present paper is to understand what implication the above-mentioned isomorphism

$$
\mathrm{CT}_{!} \simeq \mathrm{CT}_{*}^{-}
$$

has for the Eisenstein series functors Eis! and Eis $_{*}$. The conclusion will be what we will call a "strange" functional equation (0.9), explained below.

In order to explain what the "strange" functional equation does, we will need to go a little deeper into what one may call the "functional-analytic" aspects of the study of Bun $_{G}$.

0.1.4. Verdier duality on stacks. The starting point for the "analytic" issues that we will be dealing with is that the stack $\mathrm{Bun}_{G}$ is not quasi-compact (this is parallel to the fact that in the classical theory, the automorphic space is not compact, leading to a host of interesting analytic phenomena). The particular phenomenon that we will focus on is the absence of the usual Verdier duality functor, and what replaces it.

First off, it is well-known (see, e.g., [DrGa2, Sect. 2]) that if $y$ is an arbitrary reasonable $^{1}$ quasi-compact algebraic stack, then the category $D-\bmod (y)$ is compactly generated and naturally self-dual.

Perhaps, the shortest way to understand the meaning of self-duality is that the subcategory $D-\bmod (y)^{c} \subset D-\bmod (y)$ consisting of compact objects carries a canonically defined contravariant self-equivalence, called Verdier duality. A more flexible way of interpreting the same phenomenon is an equivalence, denoted $\mathbf{D} y$, between $D-\bmod (y)$ and its dual category $D-\bmod (y)^{\vee}$ (we refer the reader to [DrGa1, Sect. 1], where the basics of the notion of duality for DG categories are reviewed).

Let us now remove the assumption that $y$ be quasi-compact. Then there is another geometric condition, called "truncatability" that ensures that $D-\bmod (y)$ is compactly generated (see [DrGa2, Definition 4.1.1], where this notion is introduced). We remark here that the goal of the paper [DrGa2] was to show that the stack $\mathrm{Bun}_{G}$ is truncatable. The reader who is not familiar with this notion is advised to ignore it on the first pass.

\footnotetext{
${ }^{1}$ The word "reasonable" here does not have a technical meaning; the technical term is "QCA", which means that the automorphism group of any field-valued point is affine.
} 
Thus, let us assume that $y$ is truncatable. However, there still is no obvious replacement for Verdier duality: extending the quasi-compact case, one can define a functor

$$
\left(\mathrm{D}-\bmod (y)^{c}\right)^{\mathrm{op}} \rightarrow \mathrm{D}-\bmod (y),
$$

but it no longer lands in $D-\bmod (y)^{c}$ (unless $y$ is a disjoint union of quasi-compact stacks). In the language of dual categories, we have a functor

$$
\text { Ps-Idy,naive }: D-\bmod (y)^{\vee} \rightarrow D-\bmod (y),
$$

but it is no longer an equivalence. ${ }^{2}$

In particular, the functor Ps- $\operatorname{Id}_{\mathrm{Bun}_{G}, \text { naive }}$ is not an equivalence, unless $G$ is a torus.

0.1.5. The pseudo-identity functor. To potentially remedy this, V. Drinfeld suggested another functor, denoted

$$
\operatorname{Ps}-\operatorname{Id}_{y, !}: \mathrm{D}-\bmod (y)^{\vee} \rightarrow \mathrm{D}-\bmod (y),
$$

see [DrGa2, Sect. 4.4.8] or Sect. 3.1 of the present paper.

Now, it is not true that for all truncatable stacks $y$, the functor Ps-Idy,! is an equivalence. In [DrGa2] the stacks for which it is an equivalence are called "miraculous".

We can now formulate the main result of this paper (conjectured by V. Drinfeld):

Theorem 0.1.6. The stack $\mathrm{Bun}_{G}$ is miraculous.

We repeat that the above theorem says that the canonically defined functor Ps- $\operatorname{Id}_{\mathrm{Bun}_{G},}$ ! defines an identification of $\mathrm{D}-\bmod \left(\operatorname{Bun}_{G}\right)$ and its dual category. Equivalently, it gives rise to a (non-obvious!) contravariant self-equivalence on $\mathrm{D}-\bmod \left(\mathrm{Bun}_{G}\right)^{c}$.

0.1.7. The "strange" functional equation. Finally, we can go back and state the "strange" functional equation, which is in fact an ingredient in the proof of Theorem 0.1.6:

Theorem 0.1.8. We have a canonical isomorphism of functors

$$
\mathrm{Eis}_{!}^{-} \circ \mathrm{Ps}^{-\mathrm{Id}_{\mathrm{Bun}_{M}, !}} \simeq \mathrm{Ps}_{\mathrm{S}}-\mathrm{Id}_{\mathrm{Bun}_{G}, !} \circ\left(\mathrm{CT}_{*}\right)^{\vee} .
$$

In the Theorem 0.1.8, the functor $\left(\mathrm{CT}_{*}\right)^{\vee}$ maps

$$
\mathrm{D}-\bmod \left(\operatorname{Bun}_{M}\right)^{\vee} \rightarrow \mathrm{D}-\bmod \left(\operatorname{Bun}_{G}\right)^{\vee}
$$

and is the dual of the functor $\mathrm{CT}_{*}$. As we shall see in Sect. 1.5 , the functor $\left(\mathrm{CT}_{*}\right)^{\vee}$ is a close relative of the functor $\mathrm{Eis}_{*}$, introduced earlier.

0.2. Motivation from geometric Langlands. We shall now proceed and describe how the results of this paper fit into the geometric Langlands program. The contents of this subsection play a motivational role only, and the reader not familiar with the objects discussed below can skip this subsection and proceed to Sect. 0.3.

\footnotetext{
${ }^{2}$ The category D-mod $(y)^{\vee}$ and the functor Ps-Idy, naive will be described explicitly in Sect. 1.2.
} 
0.2.1. Statement of GLC. Let us recall the statement of the categorical geometric Langlands conjecture (GLC), according to [AG, Conjecture 10.2.2].

The left-hand (i.e., geometric) side of GLC is the DG category D-mod( $\left.\operatorname{Bun}_{G}\right)$ of D-modules on the stack $\operatorname{Bun}_{G}$.

Let $\breve{G}$ denote the Langlands dual group of $G$, and let $\operatorname{LocSys}_{\breve{G}}$ denote the (derived) stack of $\check{G}$-local systems on $X$. The right-hand (i.e., spectral) side of GLC has to do with (quasi)coherent sheaves on $\operatorname{LocSys}_{\breve{G}}$.

More precisely, In $[\mathrm{AG}]$, a certain modification of the DG category QCoh( $\left.\operatorname{LocSys}_{\breve{G}}\right)$ was introduced; we denote it by $\operatorname{IndCoh}_{\mathrm{Nilp}_{g l o b}}\left(\operatorname{LocSys}_{\breve{G}}\right)$. This category is what appears on the the spectral side of GLC.

Thus, GLC states the existence of an equivalence

$$
\mathbb{L}_{G}: \operatorname{D}-\bmod \left(\operatorname{Bun}_{G}\right) \rightarrow \operatorname{IndCoh}_{\mathrm{Nil}_{\text {glob }}}\left(\operatorname{LocSys}_{\breve{G}}\right),
$$

that satisfies a number of properties that (conjecturally) determine $\mathbb{L}_{G}$ uniquely.

The property of $\mathbb{L}_{G}$, relevant for this paper, is the compatibility of (0.2) with the functor of Eisenstein series, see Sect. 0.2.5 below.

0.2.2. Interaction of $G L C$ with duality. A feature of the spectral side crucial for this paper is that the Serre duality functor of [AG, Proposition 3.7.2] gives rise to an equivalance:

$$
\mathbf{D}_{\mathrm{LocSys}_{\breve{G}}}^{\text {Serre }}:\left(\operatorname{IndCoh}_{\mathrm{Nilp}_{\text {glob }}}\left(\operatorname{LocSys}_{\breve{G}}\right)\right)^{\vee} \rightarrow \operatorname{IndCoh}_{\mathrm{Nilp}_{\text {glob }}}\left(\operatorname{LocSys}_{\breve{G}}\right) \text {. }
$$

(Here, as in Sect. 0.1.4, for a compactly generated category $\mathbf{C}$, we denote by $\mathbf{C}^{\vee}$ the dual category.)

Hence, if we believe in the existence of an equivalence $\mathbb{L}_{G}$ of $(0.2)$, there should exist an equivalence

$$
\left(\mathrm{D}-\bmod \left(\operatorname{Bun}_{G}\right)\right)^{\vee} \simeq \mathrm{D}-\bmod \left(\operatorname{Bun}_{G}\right)
$$

Now, the pseudo-identity functor Ps- $\operatorname{Id}_{\mathrm{Bun}_{G}, !}$ mentioned in Sect. 0.1.5 and appearing in Theorem 0.1.6 is exactly supposed to perform this role. More precisely, we can enhance the statement of GLC by specifying how it is supposed to interact with duality:

Conjecture 0.2.3. The diagram

$$
\begin{aligned}
& \operatorname{D}-\bmod \left(\operatorname{Bun}_{G}\right)^{\vee} \stackrel{\left(\left(\mathbb{L}_{G}\right)^{\vee}\right)^{-1}}{\longrightarrow}\left(\operatorname{IndCoh}_{N_{i l p}}\left(\operatorname{LocSys}_{\check{G}}\right)\right)^{\vee} \\
& \downarrow \mathbf{D}_{\mathrm{LocSys}_{\breve{G}}}^{\text {Serre }} \\
& \text { Ps-Id }_{\text {Bun }_{G}, !} \downarrow \quad \operatorname{IndCoh}_{\text {Nilp }_{\text {glob }}}\left(\text { LocSys }_{\breve{G}}\right) \\
& \downarrow \tau \\
& \mathrm{D}-\bmod \left(\operatorname{Bun}_{G}\right) \stackrel{\mathbb{L}_{G}}{\longrightarrow} \quad \operatorname{IndCoh}_{\mathrm{Nilp}_{\text {glob }}}\left(\operatorname{LocSys}_{\check{G}}\right)
\end{aligned}
$$

commutes up to a cohomological shift, where $\tau$ denotes the automorphism, induced by the Cartan involution of $G$.

Remark 0.2.4. Let us comment on the presence of the Cartan involution in Conjecture 0.2.3. In fact, it can be seen already when $G$ is a torus $T$, in which case $\tau$ is the inversion automorphism.

Indeed, we let $\mathbb{L}_{T}$ be the Fourier-Mukai equivalence, and Conjecture 0.2.3 is known to hold. 
0.2.5. Interaction of $G L C$ with Eisenstein series. Let us recall (following [AG, Conjecture 12.2.9] or [Ga1, Sect. 6.4.5]) how the equivalence $\mathbb{L}_{G}$ is supposed to be compatible with the functor(s) of Eisenstein series.

For a (standard) parabolic $P \subset G$, let $\check{P}$ be the corresponding parabolic in $\check{G}$. Consider the diagram

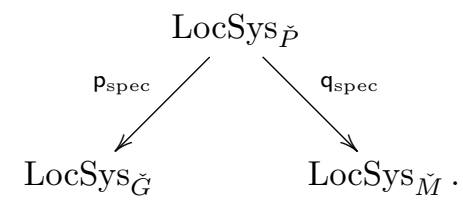

We define the functors of spectral Eisenstein series and constant term

$$
\begin{array}{ll}
\operatorname{Eis}_{\text {spec }}: \operatorname{IndCoh}\left(\operatorname{LocSys}_{\breve{M}}\right) \rightarrow \operatorname{IndCoh}\left(\operatorname{LocSys}_{\breve{G}}\right), & \operatorname{Eis}_{\text {spec }}:=\left(\mathrm{p}_{\mathrm{spec}}\right)_{*} \circ\left(\mathrm{q}_{\mathrm{spec}}\right)^{*}, \\
\mathrm{CT}_{\text {spec }}: \operatorname{IndCoh}\left(\operatorname{LocSys}_{\breve{G}}\right) \rightarrow \operatorname{IndCoh}\left(\operatorname{LocSys}_{\check{M}}\right), \quad \mathrm{CT}_{\mathrm{spec}}:=\left(\mathrm{q}_{\mathrm{spec}}\right)_{*} \circ\left(\mathrm{p}_{\mathrm{spec}}\right)^{!} .
\end{array}
$$

see $\left[\mathrm{AG}\right.$, Sect. 12.2.1] for more details. The functors $\left(\mathrm{Eis}_{\mathrm{spec}}, \mathrm{CT}_{\mathrm{spec}}\right)$ form an adjoint pair.

Remark 0.2.6. In [AG, Conjecture 12.2.9] a slightly different version of the functor Eis spec $_{\text {is }}$ given, where instead of the functor $\left(\mathrm{q}_{\mathrm{spec}}\right)^{*}$ we use $\left(\mathrm{q}_{\mathrm{spec}}\right)^{\text {! }}$. The difference between these two functors is given by tensoring by a graded line bundle on $\operatorname{LocSys}_{\breve{M}}$; this is due to the fact that the morphism $\mathrm{q}_{\mathrm{spec}}$ is Gorenstein. This difference will be immaterial for the purposes of this paper.

The compatibility of the geometric Langlands equivalence of (0.2) with Eisenstein series reads (see [AG, Conjecture 12.2.9]):

Conjecture 0.2.7. The diagram

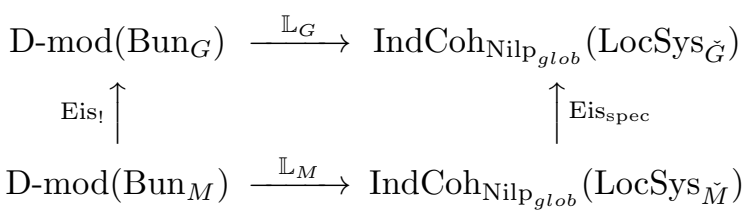

commutes up to an automorphism of $\operatorname{IndCoh}_{\text {Nilp }_{\text {glob }}}\left(\operatorname{LocSys}_{\check{M}}\right)$, given by tensoring with a certain canonically defined graded line bundle on LocSys $_{\check{M}}$.

0.2.8. Recovering the "strange" functional equation. Let us now analyze what the combination of Conjectures 0.2.3 and 0.2.7 says about the interaction of the functor Ps- $\operatorname{Id}_{\mathrm{Bun}_{G}, !}$ ! with Eis! The conclusion that we will draw will amount to Theorem 0.1 .8 of the present paper (the reader may safely choose to skip the derivation that follows).

First, passing to the right adjoint and then dual functors in (0.6), we obtain a diagram

$$
\begin{array}{cc}
\operatorname{D}-\bmod \left(\operatorname{Bun}_{G}\right)^{\vee} \stackrel{\left(\mathbb{L}_{G}^{\vee}\right)^{-1}}{\longrightarrow} & \left(\operatorname{IndCoh}_{\text {Nilp }_{\text {glob }}}\left(\operatorname{LocSys}_{\check{G}}\right)^{\vee}\right. \\
\left(\mathrm{CT}_{*}\right)^{\vee} \uparrow & \uparrow\left(\mathrm{CT}_{\mathrm{spec}}\right)^{\vee} \\
\operatorname{D-mod}\left(\operatorname{Bun}_{M}\right)^{\vee} \stackrel{\left(\mathbb{L}_{M}^{\vee}\right)^{-1}}{\longrightarrow}\left(\operatorname{IndCoh}_{\mathrm{Nilp}_{\text {glob }}}\left(\operatorname{LocSys}_{\check{M}}\right)\right)^{\vee}
\end{array}
$$

that commutes up to a tensoring by a graded line bundle on $\operatorname{LocSys}_{\check{M}}$. 
Next, we note that the diagram

$$
\begin{aligned}
& \left(\operatorname{IndCoh}_{\mathrm{Nilp}_{\text {glob }}}\left(\operatorname{LocSys}_{\breve{G}}\right)^{\vee} \stackrel{\text { D. }_{\mathrm{LocSys}_{\breve{G}}}^{\text {Serre }}}{\longrightarrow} \operatorname{IndCoh}_{\mathrm{Nilp}_{\text {glob }}}\left(\operatorname{LocSys}_{\breve{G}}\right)\right. \\
& \left(\mathrm{CT}_{\text {spec }}\right)^{\vee} \uparrow \quad \mathrm{Eis}_{\text {spec }} \uparrow \\
& \left(\operatorname{IndCoh}_{\mathrm{Nilp}_{\text {glob }}}\left(\operatorname{LocSys}_{\check{M}}\right)^{\vee} \stackrel{\mathrm{D}_{\mathrm{LocSys}_{\check{M}}}^{\text {Serre }}}{\longrightarrow} \operatorname{IndCoh}_{\mathrm{Nilp}_{g l o b}}\left(\operatorname{LocSys}_{\check{M}}\right)\right.
\end{aligned}
$$

also commutes up to a tensoring by a graded line bundle on LocSys $\check{M}$, see Remark 0.2.6.

Now, juxtaposing the diagrams (0.6), (0.7), (0.8) with the diagrams (0.4) for the groups $G$ and $M$ respectively, we obtain a commutative diagram:

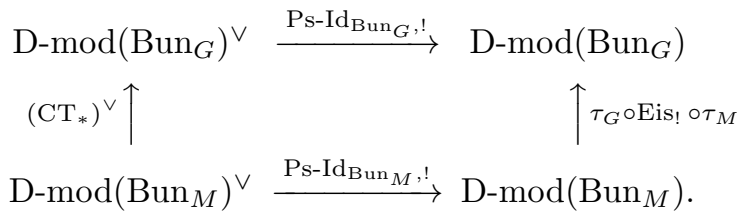

Notice now that $\tau_{G} \circ$ Eis! $\circ \tau_{M} \simeq$ Eis! $_{!}^{-}$, so the commutative diagram (0.9) recovers the isomorphism of Theorem 0.1.8.

0.3. The usual functional equation. As was mentioned above, we view the commutativity of the diagram (0.9) as a kind of "strange" functional equation, hence the title of this paper.

Let us now compare it to the usual functional equation of [BG, Theorem 2.1.8].

0.3.1. In loc.cit. one considered the case of $P=B$, the Borel subgroup and hence $M=T$, the abstract Cartan. We consider the full subcategory

$$
\mathrm{D}-\bmod \left(\operatorname{Bun}_{T}\right)^{\mathrm{reg}} \subset \mathrm{D}-\bmod \left(\operatorname{Bun}_{T}\right),
$$

defined as in [BG, Sect. 2.1.7]. This is a full subcategory that under the Fourier-Mukai equivalence

$$
\mathrm{D}-\bmod \left(\operatorname{Bun}_{T}\right) \simeq \operatorname{QCoh}\left(\operatorname{LocSys}_{\breve{T}}\right)
$$

corresponds to

$$
\mathrm{QCoh}\left(\operatorname{LocSys}_{\check{T}}^{\mathrm{reg}}\right) \hookrightarrow \mathrm{QCoh}^{\mathrm{e}}\left(\operatorname{LocSys}_{\check{T}}\right),
$$

where $\operatorname{LocSys}_{\breve{T}}^{\text {reg }} \subset \operatorname{LocSys}_{\breve{T}}$ is the open locus of $\operatorname{LocSys}_{\breve{T}}$ consisting of those $\check{T}$-local systems that for every root $\alpha$ of $\check{T}$ induce a non-trivial local system for $\mathbb{G}_{m}$.

Instead of the functor Eis! , or the functor that we introduce as Eis ${ }_{*}:=p_{*} \circ q^{!}$(see Sect. 1.1.6), an intermediate version was considered in [BG, Sect. 2.1], which we will denote here by Eis!* . The definition of Eis!* uses the compactification of the morphism p, introduced in [BG, Sect. $1.2]$ :

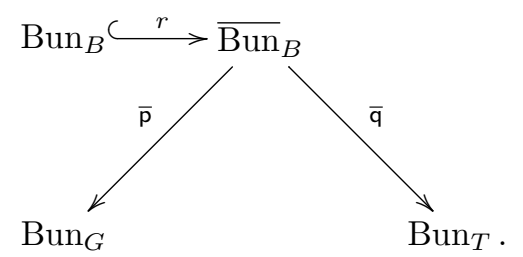

The assertion of [BG, Theorem 2.1.8] (for the longest element of the Weyl group) is: 
Theorem 0.3.2. The following diagram of functors

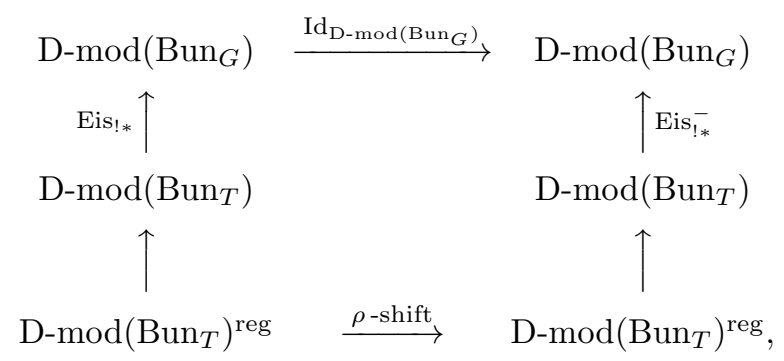

commutes up to a cohomological shift, where $\rho$-shift is the functor of translation by the point $2 \rho\left(\Omega_{X}\right){ }^{3}$

Theorem 0.3.2 is a geometric analog of the usual functional equation for Eisenstein series in the theory of automorphic functions.

0.3.3. Let us emphasize the following points of difference between Theorems 0.1.8 and 0.3.2:

- Theorem 0.1.8 compares the functors Eis! and $\left(\mathrm{CT}_{*}\right)^{\vee}$ that take values in different categories, i.e., $\mathrm{D}-\bmod \left(\operatorname{Bun}_{G}\right)$ vs. $\mathrm{D}-\bmod \left(\operatorname{Bun}_{G}\right)^{\vee}$, whereas in Theorem 0.3.2 both Eis!* and Eis $_{!_{*}}^{-}$map to D-mod $\left(\operatorname{Bun}_{G}\right)$.

- The vertical arrows in Theorem 0.1.8 use geometrically different functors, while in Theorem 0.3.2 these are functors of the same nature, i.e., Eis - $_{\text {!* }}$ and Eis $_{!_{*}}^{-}$.

- The upper horizontal arrow Theorem 0.1.8 is the geometrically non-trivial functor $\mathrm{Ps}-\mathrm{Id}_{\mathrm{Bun}_{G}, !}$, while in Theorem 0.3.2 it is the identity functor.

- The lower horizontal arrow in Theorem 0.1.8 for $M=T$ is isomorphic to the identity functor, up to a cohomological shift, while in Theorem 0.3.2 we have the functor of $\rho$-shift.

- The commutation in 0.1.8 takes place on all of $\mathrm{D}-\bmod \left(\operatorname{Bun}_{T}\right)$, whereas in Theorem 0.3.2, it only takes place on $\mathrm{D}-\bmod \left(\mathrm{Bun}_{T}\right)^{\mathrm{reg}}$.

0.4. Interaction with cuspidality. There is yet one more set of results contained in this paper, which has to do with the notion of cuspidality.

0.4.1. The cuspidal subcategories

$$
\mathrm{D}-\bmod \left(\operatorname{Bun}_{G}\right)_{\text {cusp }} \subset \mathrm{D}-\bmod \left(\operatorname{Bun}_{G}\right) \text { and }\left(\mathrm{D}-\bmod \left(\operatorname{Bun}_{G}\right)^{\vee}\right)_{\text {cusp }} \subset \mathrm{D}-\bmod \left(\operatorname{Bun}_{G}\right)^{\vee}
$$

are defined as right-orthogonals of the subcategories generated by the essential images of the functors

Eis! : D-mod $\left(\operatorname{Bun}_{M}\right) \rightarrow \mathrm{D}-\bmod \left(\operatorname{Bun}_{G}\right)$ and $\left(\mathrm{CT}_{*}\right)^{\vee}: \mathrm{D}-\bmod \left(\operatorname{Bun}_{M}\right)^{\vee} \rightarrow \mathrm{D}-\bmod \left(\operatorname{Bun}_{G}\right)^{\vee}$, respectively, for all proper parabolics $P$ of $G$.

0.4.2. Let us return to the setting of Sect. 0.1.4 are recall the "naive" functor

$$
\mathrm{Ps}_{\mathrm{S}} \mathrm{Id}_{\mathrm{Bun}_{G}, \text { naive }}: \mathrm{D}-\bmod \left(\operatorname{Bun}_{G}\right)^{\vee} \rightarrow \mathrm{D}-\bmod \left(\operatorname{Bun}_{G}\right),
$$

see Sect. 0.1.4.

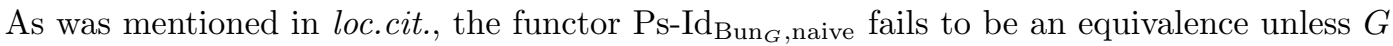
is a torus. However, in Theorem 2.2.7 we show:

\footnotetext{
${ }^{3}$ Here $2 \rho: \mathbb{G}_{m} \rightarrow T$ is the coweight equal to the sum of positive coroots, and $\Omega_{X} \in \operatorname{Pic}(X)=\operatorname{Bun}_{\mathbb{G}_{m}}$ is the canonical line bundle on $X$.
} 
Theorem 0.4.3. The restriction of the functor $\mathrm{Ps}_{\mathrm{s}} \mathrm{Id}_{\mathrm{Bun}}$, naive $t o$

$$
\left(\mathrm{D}-\bmod \left(\operatorname{Bun}_{G}\right)^{\vee}\right)_{\text {cusp }} \subset \mathrm{D}-\bmod \left(\operatorname{Bun}_{G}\right)^{\vee}
$$

defines an equivalence

$$
\left(\mathrm{D}-\bmod \left(\operatorname{Bun}_{G}\right)^{\vee}\right)_{\text {cusp }} \rightarrow \mathrm{D}-\bmod \left(\operatorname{Bun}_{G}\right)_{\text {cusp }} .
$$

One can view Theorem 0.4.3 as expressing the fact that the objects of $\left(\mathrm{D}-\bmod \left(\mathrm{Bun}_{G}\right)^{\vee}\right)_{\text {cusp }}$ and D-mod $\left(\operatorname{Bun}_{G}\right)_{\text {cusp }}$ are "supported" on quasi-compact open substacks (see Propositions 2.3.2 and 2.3.4 for a precise statement).

0.4.4. In addition, in Corollary 3.3.2 we show:

Theorem 0.4.5. The functors

$$
\operatorname{Ps}-\left.\operatorname{Id}_{\mathrm{Bun}_{G}, \text { naive }}\right|_{\left(\mathrm{D}-\bmod \left(\operatorname{Bun}_{G}\right)^{\vee}\right)_{\text {cusp }}} \text { and } \mathrm{Ps}-\left.\operatorname{Id}_{\mathrm{Bun}_{G}, !}\right|_{\left(\mathrm{D}-\bmod \left(\operatorname{Bun}_{G}\right)^{\vee}\right)_{\text {cusp }}}
$$

are isomorphic up to a cohomological shift.

Theorem 0.4.5 is responsible for the fact that previous studies in geometric Langlands correspondence that involved only cuspidal objects did not see the appearance of the functor Ps-Id $\mathrm{Bun}_{G}$ ! and one could afford to ignore the difference between D-mod(Bun $\left.{ }_{G}\right)$ and $\mathrm{D}-\bmod \left(\operatorname{Bun}_{G}\right)^{\vee}$. In other words, usual manipulations with Verdier duality on cuspidal objects did not produce wrong results.

\subsection{Structure of the paper.}

0.5.1. In Sect. 1 we recall the setting of [DrGa3], and list the various Eisenstein series and constant term functors for the usual category $\mathrm{D}-\bmod \left(\operatorname{Bun}_{G}\right)$. In fact there are two adjoint pairs: $\left(\right.$ Eis!, $\left.\mathrm{CT}_{*}\right)$ and $\left(\mathrm{CT}_{!}^{\mu}, \mathrm{Eis}_{*}^{\mu}\right)$, where in the latter pair the superscript $\mu \in \pi_{1}(M)=\pi_{0}\left(\operatorname{Bun}_{M}\right)$ indicates that we are considering one connected component of $\operatorname{Bun}_{M}$ at a time.

We recall the main result of [DrGa3] that says that the functors $\mathrm{CT}_{*}$ and $\mathrm{CT}_{!}^{-}$are canonically isomorphic.

Next, we consider the category $\mathrm{D}-\bmod \left(\mathrm{Bun}_{G}\right)_{\mathrm{co}}$, which is nearly tautologically identified with the category that we have so far denoted $\mathrm{D}-\bmod \left(\operatorname{Bun}_{G}\right)^{\vee}$, and introduce the corresponding Eisenstein series and constant term functors:

$$
\left(\mathrm{Eis}_{\mathrm{co}, *}, \mathrm{CT}_{\mathrm{co}, ?)}\right) \text { and }\left(\mathrm{CT}_{\mathrm{co}, *}^{\mu}, \operatorname{Eis}_{\mathrm{co}, ?}^{\mu}\right),
$$

where $\operatorname{Eis}_{\mathrm{co} *}:=\left(\mathrm{CT}_{*}\right)^{\vee}, \mathrm{CT}_{\mathrm{co}, *}^{\mu}:=\left(\operatorname{Eis}_{*}^{\mu}\right)^{\vee}$.

The functor $\mathrm{CT}_{\mathrm{co}, \text { ? }}$ is something that we do not know how to express in terms of the usual functors in the theory of D-modules; it can be regarded as a non-standard functor in the terminology of [DrGa2, Sect. 3.3].

A priori, the functor $\mathrm{Eis}_{\mathrm{co}, ?}^{\mu}$ would also be a non-standard functor. However, the isomorphism $\mathrm{CT}_{*} \simeq \mathrm{CT}_{!}^{-}$gives rise to an isomorphism

$$
\operatorname{Eis}_{\mathrm{co}, ?} \simeq \operatorname{Eis}_{\mathrm{co}, *}^{-} .
$$


0.5.2. In Sect. 2 we recall the definition of the functor

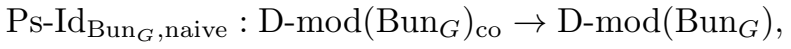

and show that it intertwines the functors $\mathrm{Eis}_{\mathrm{co}, *}$ and $\mathrm{Eis}_{*}$, and $\mathrm{CT}_{\mathrm{co}, *}$ and $\mathrm{CT}_{*}$, respectively.

The remainder of this section is devoted to the study of the subcategory

$$
\mathrm{D}-\bmod \left(\operatorname{Bun}_{G}\right)_{\text {co,cusp }} \subset \mathrm{D}-\bmod \left(\operatorname{Bun}_{G}\right) \text {, }
$$

and the proof of Theorem 0.4.3, which says that the functor Ps- $\mathrm{Id}_{\mathrm{Bun}_{G} \text {,naive }}$ defines an equivalence from D-mod $\left(\operatorname{Bun}_{G}\right)_{\text {co,cusp }}$ to $\mathrm{D}-\bmod \left(\operatorname{Bun}_{G}\right)_{\text {cusp }} \subset \mathrm{D}-\bmod \left(\operatorname{Bun}_{G}\right)$.

0.5.3. In Sect. 3 we introduce the functor

$$
\operatorname{Ps}_{-} \operatorname{Id}_{B_{G}, !}: \mathrm{D}-\bmod \left(\operatorname{Bun}_{G}\right)_{\text {co }} \rightarrow \mathrm{D}-\bmod \left(\operatorname{Bun}_{G}\right),
$$

and study its behavior vis-à-vis the functor Ps- $\mathrm{Id}_{\mathrm{Bun}}$, naive. The relation is expressed by Proposition 3.2.6, whose proof is deferred to [Sch]. Proposition 3.2.6 essentially says that the difference between Ps-Id $\mathrm{Bun}_{G}$, ! and $\mathrm{Ps}_{\mathrm{S}}-\mathrm{Id}_{\mathrm{Bun}_{G} \text {,naive }}$ can be expressed in terms of the Eisenstein and constant term functors for proper parabolics.

We prove Theorem 0.4.5 that says that the functors $\mathrm{Ps}_{\mathrm{S}}-\mathrm{Id}_{\mathrm{Bun}}$, ! and $\mathrm{Ps}_{\mathrm{s}} \mathrm{Id}_{\mathrm{Bun}}$, naive are isomorphic (up to a cohomological shift), when evaluated on cuspidal objects.

0.5.4. In Sect. 4 we prove our "strange" functional equation, i.e., Theorem 0.1.8. The proof is basically a formal manipulation from the isomorphism $\mathrm{CT}_{*} \simeq \mathrm{CT}_{!}^{-}$.

Having Theorem 0.1.8, we get control of the behavior of the functor Ps-Id $\mathrm{Bun}_{G}$, ! on the Eisenstein part of the category $\mathrm{D}-\bmod \left(\operatorname{Bun}_{G}\right)_{\text {co }}$. From here we deduce our main result, Theorem 0.1.6.

0.6. Conventions. The conventions in this paper follow those adopted in [DrGa2]. We refer the reader to loc.cit. for a review of the theory of DG categories (freely used in this paper), and the theory of D-modules on stacks.

0.7. Acknowledgements. The author would like to express his gratitude to V. Drinfeld. The main results of this paper, in particular, Theorem 0.1.8, were obtained in collaboration with him.

Even more crucially, the very idea of the pseudo-identity functor Ps- $\operatorname{Id}_{\mathrm{Bun}_{G}, !}$ is the invention of his.

The author is supported by NSF grant DMS-1063470.

\section{The InVEntory OF CATEGORIES AND FUnCTORS}

\subsection{Eisenstein series and constant term functors.}

1.1.1. Let $P$ be a parabolic in $G$ with Levi quotient $M$. For

$$
\mu \in \pi_{1}(M) \simeq \pi_{0}\left(\operatorname{Bun}_{M}\right) \simeq \pi_{0}\left(\operatorname{Bun}_{P}\right),
$$

let $\operatorname{Bun}_{M}^{\mu}$ (resp., $\operatorname{Bun}_{P}^{\mu}$ ) denote the corresponding connected component of $\operatorname{Bun}_{M}($ resp., Bun $P$ ). 
1.1.2. Consider the diagram

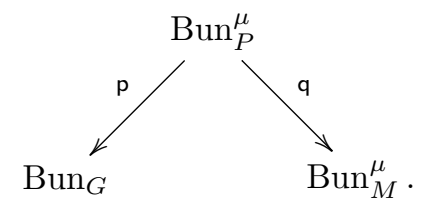

We consider the functor

$$
\mathrm{CT}_{*}^{\mu}: \mathrm{D}-\bmod \left(\mathrm{Bun}_{G}\right) \rightarrow \mathrm{D}-\bmod \left(\mathrm{Bun}_{M}^{\mu}\right), \quad \mathrm{CT}_{*}^{\mu}=\mathrm{q}_{*} \circ \mathrm{p} !
$$

1.1.3. According to [DrGa3, Corollary 1.1.3], the functor $\mathrm{CT}_{*}^{\mu}$ admits a left adjoint, denoted by Eis $_{!}^{\mu}$. Explicitly,

$$
\operatorname{Eis}_{!}^{\mu}=\mathrm{p}_{!} \circ \mathrm{q}^{*} .
$$

The above expression has to be understood as follows: the functor

$$
\mathrm{q}^{*}: \mathrm{D}-\bmod \left(\operatorname{Bun}_{M}^{\mu}\right) \rightarrow \mathrm{D}-\bmod \left(\operatorname{Bun}_{P}^{\mu}\right)
$$

is defined (because the morphism $q$ is smooth), and the partially defined functor $p_{!}$, left adjoint to $\mathrm{p}^{!}$, is defined on the essential image of $\mathrm{q}^{*}$ by [DrGa3, Proposition 1.1.2].

1.1.4. We define the functor $\mathrm{CT}_{*}: \mathrm{D}-\bmod \left(\mathrm{Bun}_{G}\right) \rightarrow \mathrm{D}-\bmod \left(\mathrm{Bun}_{M}\right)$ as

$$
\mathrm{CT}_{*} \simeq \bigoplus_{\mu} \mathrm{CT}_{*}^{\mu}
$$

We define the functor Eis! : D-mod( $\left.\operatorname{Bun}_{M}\right) \rightarrow \mathrm{D}-\bmod \left(\operatorname{Bun}_{G}\right)$ as

$$
\text { Eis! } \simeq \bigoplus_{\mu} \operatorname{Eis}_{!}^{\mu} .
$$

Lemma 1.1.5. The functor Eis! is the left adjoint of $\mathrm{CT}_{*}$.

Proof. Follows from the fact that

$$
\bigoplus_{\mu} \mathrm{CT}_{*}^{\mu} \simeq \prod_{\mu} \mathrm{CT}_{*}^{\mu}
$$

1.1.6. We now consider the functor $\operatorname{Eis}_{*}^{\mu}: \mathrm{D}-\bmod \left(\operatorname{Bun}_{M}^{\mu}\right) \rightarrow \mathrm{D}-\bmod \left(\operatorname{Bun}_{G}\right)$, defined as

$$
\operatorname{Eis}_{*}^{\mu}=\mathrm{p}_{*} \circ \mathrm{q}^{!} .
$$

We let $\mathrm{Eis}_{*}^{\mu,-}$ and $\mathrm{CT}_{*}^{\mu,-}$ be similarly defined functors when instead of $P$ we use the opposite parabolic $P^{-}$(we identify the Levi quotients of $P$ and $P^{-}$via the isomorphsim $M \simeq P \cap P^{-}$).

The following is the main result of [DrGa3]:

Theorem 1.1.7. The functor Eis $_{*}^{\mu}$ canonically identifies with the right adjoint of $\mathrm{CT}_{*}^{\mu,-}$. 
1.1.8. We will use the notation

$$
\mathrm{CT}_{!}^{\mu}: \mathrm{D}-\bmod \left(\operatorname{Bun}_{G}\right) \rightarrow \mathrm{D}-\bmod \left(\operatorname{Bun}_{M}^{\mu}\right)
$$

for the left adjoint of $\operatorname{Eis}_{*}^{\mu}$. If $\mathcal{F} \in \mathrm{D}-\bmod \left(\operatorname{Bun}_{G}\right)$ is such that the partially defined left adjoint $\mathrm{p}^{*}$ of $\mathrm{p}_{*}$ is defined on $\mathcal{F}$, then we have

$$
\mathrm{CT}_{!}^{\mu}(\mathcal{F}) \simeq \mathrm{q} ! \circ \mathrm{p}^{*}(\mathcal{F}) .
$$

(The functor q!, left adjoint to q!, is well-defined by [DrGa3, Sect. 3.1.5].)

Hence, Theorem 1.1.7 can be reformulated as saying that $\mathrm{CT}_{!}^{\mu}$ exists and is canonically isomorphic to $\mathrm{CT}_{*}^{\mu,-}$.

1.1.9. We define the functor $\mathrm{CT}_{!}: \mathrm{D}-\bmod \left(\mathrm{Bun}_{G}\right) \rightarrow \mathrm{D}-\bmod \left(\mathrm{Bun}_{M}\right)$ as

$$
\mathrm{CT}_{!} \simeq \bigoplus_{\mu} \mathrm{CT}_{!}^{\mu}
$$

so $\mathrm{CT}_{!} \simeq \mathrm{CT}_{*}^{-}$.

We define the functor $\operatorname{Eis}_{*}: \mathrm{D}-\bmod \left(\operatorname{Bun}_{M}\right) \rightarrow \mathrm{D}-\bmod \left(\operatorname{Bun}_{G}\right)$ as

$$
\operatorname{Eis}_{*} \simeq \bigoplus_{\mu} \operatorname{Eis}_{*}^{\mu} .
$$

We note, however, that it is no longer true that $\mathrm{Eis}_{*}$ is the right adjoint of $\mathrm{CT}_{\text {! }}$ (Rather, the right adjoint of $\mathrm{CT}_{!}$is the functor $\prod_{\mu} \mathrm{Eis}_{*}^{\mu}$.)

In fact, one can show that the functor Eis $_{*}$ does not admit a left adjoint, see [DrGa3, Sect. 1.2.1].

\subsection{The dual category.}

1.2.1. Let op-qc $(G)$ denote the poset of open substacks $U \stackrel{j}{\rightarrow} \operatorname{Bun}_{G}$ such that the intersection of $U$ with every connected component of $\operatorname{Bun}_{G}$ is quasi-compact.

We have

$$
\mathrm{D}-\bmod \left(\operatorname{Bun}_{G}\right) \simeq \lim _{U \in \mathrm{op}-\mathrm{qc}(G)} \operatorname{D}-\bmod (U)
$$

where for $U_{1} \stackrel{j_{1,2}}{\hookrightarrow} U_{2}$, the corresponding functor $\mathrm{D}-\bmod \left(U_{2}\right) \rightarrow \mathrm{D}-\bmod \left(U_{1}\right)$ is $j_{1,2}^{*}$ (see, e.g., [DrGa2, Lemma 2.3.2] for the proof).

Under the equivalence (1.2), for

$$
\left(U \stackrel{j}{\hookrightarrow} \operatorname{Bun}_{G}\right) \in \operatorname{op}-q c(G),
$$

the tautological evaluation functor $\mathrm{D}-\bmod \left(\operatorname{Bun}_{G}\right) \rightarrow \mathrm{D}-\bmod (U)$ is $j^{*}$. 
D. GAITSGORY

1.2.2. The following DG category was introduced in [DrGa2, Sect. 4.3.3]:

$$
\mathrm{D}-\bmod \left(\operatorname{Bun}_{G}\right)_{\mathrm{co}}:=\underset{U \in \mathrm{op}-\mathrm{qc}(G)}{\operatorname{colim}} \operatorname{D-mod}(U),
$$

where for $U_{1} \stackrel{j_{1,2}}{\longrightarrow} U_{2}$, the corresponding functor $\mathrm{D}-\bmod \left(U_{1}\right) \rightarrow \mathrm{D}-\bmod \left(U_{2}\right)$ is $\left(j_{1,2}\right)_{*}$, and where the colimit is taken in the category of cocomplete DG categories and continuous functors.

For $\left(U \stackrel{j}{\hookrightarrow} \operatorname{Bun}_{G}\right) \in \operatorname{op}-\mathrm{qc}(G)$ we let $j_{\mathrm{co}, *}$ denote the tautological functor

$$
j_{\mathrm{co}, *}: \mathrm{D}-\bmod (U) \rightarrow \mathrm{D}-\bmod \left(\operatorname{Bun}_{G}\right)_{\mathrm{co}} .
$$

1.2.3. Verdier duality functors

$$
\mathbf{D}_{U}: \mathrm{D}-\bmod (U)^{\vee} \simeq \mathrm{D}-\bmod (U)
$$

for $U \in \mathrm{op}-\mathrm{qc}(G)$ and the identifications

$$
\left(\left(j_{1,2}\right)_{*}\right)^{\vee} \simeq\left(j_{1,2}\right)^{*}, \quad U_{1} \stackrel{j_{1,2}}{\hookrightarrow} U_{2}
$$

give rise to an identification

$$
\text { Funct }_{\text {cont }}\left(\mathrm{D}-\bmod \left(\operatorname{Bun}_{G}\right)_{\mathrm{co}}, \mathrm{Vect}\right) \simeq \mathrm{D}-\bmod \left(\operatorname{Bun}_{G}\right) .
$$

Now, the main result of [DrGa2], namely, Theorem 4.1.8, implies:

Theorem 1.2.4. The category $\mathrm{D}-\bmod \left(\mathrm{Bun}_{G}\right)_{\mathrm{co}}$ is compactly generated (and, in particular, dualizable).

Proof. The truncatability of $\operatorname{Bun}_{G}$ means that in the presentation of D-mod $\left(\operatorname{Bun}_{G}\right)_{\text {co }}$ as a colimit (1.3), we can replace the index poset op-qc $(G)$ by a cofinal poset that consists of quasicompact open substacks that are co-truncative.

Then the resulting colimit

$$
\underset{U}{\operatorname{colim}} \mathrm{D}-\bmod (U)
$$

consists of compactly generated categories and functors that preserve compactness. In this case, the resulting colimit category is compactly generated, e.g., by [DrGa2, Corollary 1.9,4].

From (1.5), and knowing that $\mathrm{D}-\bmod \left(\operatorname{Bun}_{G}\right)_{\mathrm{co}}$ is dualizable, we obtain a canonical identification

$$
\mathbf{D}_{\mathrm{Bun}_{G}}: \mathrm{D}-\bmod \left(\operatorname{Bun}_{G}\right)^{\vee} \simeq \mathrm{D}-\bmod \left(\operatorname{Bun}_{G}\right)_{\mathrm{co}} .
$$

Under this identification, for $\left(U \stackrel{j}{\hookrightarrow} \operatorname{Bun}_{G}\right) \in$ op-qc $(G)$ we have the following canonical identification of functors

$$
\left(j_{\mathrm{co}, *}\right)^{\vee} \simeq j^{*}
$$

1.2.5. Similar constructions and notation apply when instead of all of $\operatorname{Bun}_{G}$ we consider one of its connected components $\operatorname{Bun}_{G}^{\lambda}, \lambda \in \pi_{1}(G)$.

\subsection{Dual, adjoint and conjugate functors.}

1.3.1. Let $\mathbf{C}_{1}$ and $\mathbf{C}_{2}$ be two DG categories, and let

$$
\mathrm{F}: \mathbf{C}_{1} \rightleftarrows \mathbf{C}_{2}: \mathrm{G}
$$

be a pair of continuous mutually adjoint functors. 
1.3.2. By passing to dual functors, the adjunction data

$$
\mathrm{Id}_{\mathbf{C}_{1}} \rightarrow \mathrm{G} \circ \mathrm{F} \text { and } \mathrm{F} \circ \mathrm{G} \rightarrow \operatorname{Id}_{\mathbf{C}_{2}}
$$

gives rise to

making

$$
\operatorname{Id}_{\mathbf{C}_{1}^{\vee}} \rightarrow \mathrm{F}^{\vee} \circ \mathrm{G}^{\vee} \text { and } \mathrm{G}^{\vee} \circ \mathrm{F}^{\vee} \rightarrow \operatorname{Id}_{\mathbf{C}_{2}^{\vee}},
$$

$$
\mathrm{G}^{\vee}: \mathbf{C}_{1}^{\vee} \rightleftarrows \mathbf{C}_{2}^{\vee}: \mathrm{F}^{\vee}
$$

into a pair of adjoint functors.

1.3.3. Assume now that $\mathbf{C}_{1}$ is compactly generated. In this case, the fact that the right adjoint $G$ of $F$ is continuous is equivalent to the fact that $F$ preserves compactness. I.e., it defines a functor between non-cocomplete DG categories

$$
\mathbf{C}_{1}^{c} \rightarrow \mathbf{C}_{2}^{c},
$$

and hence, by passing to the opposite categories, a functor

$$
\left(\mathbf{C}_{1}^{c}\right)^{\mathrm{op}} \rightarrow\left(\mathbf{C}_{2}^{c}\right)^{\mathrm{op}}
$$

Following [Ga2, Sect. 1.5], we let

$$
\mathrm{F}^{\mathrm{op}}: \mathbf{C}_{1}^{\vee} \rightarrow \mathbf{C}_{2}^{\vee}
$$

denote the functor obtained as the composition of:

(i) The identification $\mathbf{C}_{1}^{\vee} \simeq \operatorname{Ind}\left(\left(\mathbf{C}_{1}^{c}\right)^{\mathrm{op}}\right)$;

(ii) The ind-extension $\operatorname{Ind}\left(\left(\mathbf{C}_{1}^{c}\right)^{\mathrm{op}}\right) \rightarrow \operatorname{Ind}\left(\left(\mathbf{C}_{2}^{c}\right)^{\mathrm{op}}\right)$ of $(1.7)$;

(iii) The fully faithful embedding $\left(\mathbf{C}_{2}^{c}\right)^{\mathrm{op}} \hookrightarrow \mathbf{C}_{2}^{\vee}$.

We call $\mathrm{F}^{\mathrm{op}}$ the functor conjugate to $\mathrm{F}$.

1.3.4. The following is [Ga2, Lemma 1.5.3]:

Lemma 1.3.5. We have a canonical isomorphism of functors $\mathrm{F}^{\mathrm{op}} \simeq \mathrm{G}^{\vee}$.

\subsection{Dual Eisenstein series and constant term functors.}

1.4.1. We define the functor

$$
\operatorname{Eis}_{\mathrm{co}, *}^{\mu}: \mathrm{D}-\bmod \left(\operatorname{Bun}_{M}^{\mu}\right)_{\mathrm{co}} \rightarrow \mathrm{D}-\bmod \left(\operatorname{Bun}_{G}\right)_{\mathrm{co}}
$$

as

$$
\operatorname{Eis}_{\mathrm{co}, *}^{\mu} \simeq\left(\mathrm{CT}_{*}^{\mu}\right)^{\vee}
$$

under the identifications (1.6) and

$$
\mathbf{D}_{\mathrm{Bun}_{M}^{\mu}}: \mathrm{D}-\bmod \left(\operatorname{Bun}_{M}^{\mu}\right)^{\vee} \simeq \mathrm{D}-\bmod \left(\operatorname{Bun}_{M}^{\mu}\right)_{\mathrm{co}} .
$$

We define

$$
\operatorname{Eis}_{\mathrm{co}, *}: \mathrm{D}-\bmod \left(\operatorname{Bun}_{M}\right)_{\text {co }} \rightarrow \mathrm{D}-\bmod \left(\operatorname{Bun}_{G}\right)_{\mathrm{co}}
$$

as

$$
\operatorname{Eis}_{\mathrm{co}, *}:=\bigoplus_{\mu} \operatorname{Eis}_{\mathrm{co}, *}^{\mu} \simeq\left(\mathrm{CT}_{*}\right)^{\vee} .
$$

Note that by Lemma 1.3.5, we have:

Corollary 1.4.2. There are canonical isomorphisms

$$
\operatorname{Eis}_{\mathrm{co}, *} \simeq\left(\text { Eis! }_{!}\right)^{\mathrm{op}} \text { and } \operatorname{Eis}_{\mathrm{co}, *}^{\mu} \simeq\left(\operatorname{Eis}_{!}^{\mu}\right)^{\mathrm{op}} .
$$


1.4.3. We define the functor

$$
\mathrm{CT}_{\mathrm{co}, *}^{\mu}: \mathrm{D}-\bmod \left(\operatorname{Bun}_{G}\right)_{\mathrm{co}} \rightarrow \mathrm{D}-\bmod \left(\mathrm{Bun}_{M}^{\mu}\right)_{\mathrm{co}}
$$

as

$$
\mathrm{CT}_{\mathrm{co}, *}^{\mu} \simeq\left(\operatorname{Eis}_{*}^{\mu}\right)^{\vee}
$$

We define

$$
\mathrm{CT}_{\mathrm{co}, *}: \mathrm{D}-\bmod \left(\operatorname{Bun}_{G}\right)_{\mathrm{co}} \rightarrow \mathrm{D}-\bmod \left(\operatorname{Bun}_{M}\right)_{\mathrm{co}}
$$

as

$$
\mathrm{CT}_{\mathrm{co}, *}:=\bigoplus_{\mu} \mathrm{CT}_{\mathrm{co}, *}^{\mu} \simeq\left(\operatorname{Eis}_{*}\right)^{\vee}
$$

From Lemma 1.3.5, we obtain:

Corollary 1.4.4. There is a canonical isomorphism

$$
\mathrm{CT}_{\mathrm{co}, *}^{\mu} \simeq\left(\mathrm{CT}_{!}^{\mu}\right)^{\mathrm{op}} \text {. }
$$

1.4.5. Define also

$$
\begin{aligned}
& \mathrm{CT}_{\mathrm{co}, ?}^{\mu}:=\left(\operatorname{Eis}_{!}^{\mu}\right)^{\vee} \text { and } \mathrm{CT}_{\mathrm{co}, ?}:=\left(\mathrm{Eis}_{!}\right)^{\vee} \simeq \bigoplus_{\mu} \mathrm{CT}_{\mathrm{co}, ?}^{\mu}, \\
& \operatorname{Eis}_{\mathrm{co}, ?}^{\mu}:=\left(\mathrm{CT}_{!}^{\mu}\right)^{\vee} \text { and } \operatorname{Eis}_{\mathrm{co}, ?}:=\left(\mathrm{CT}_{!}\right)^{\vee} \simeq \bigoplus_{\mu} \operatorname{Eis}_{\mathrm{co}, ?}^{\mu} .
\end{aligned}
$$

By Sect. 1.3.2, we obtain the following pairs of adjoint functors

$$
\begin{aligned}
& \operatorname{Eis}_{\mathrm{co}, *}^{\mu}: \mathrm{D}-\bmod \left(\operatorname{Bun}_{M}^{\mu}\right)_{\mathrm{co}} \rightleftarrows \mathrm{D}-\bmod \left(\operatorname{Bun}_{G}\right)_{\mathrm{co}}: \mathrm{CT}_{\mathrm{co}, ?}^{\mu}, \\
& \operatorname{Eis}_{\mathrm{co}, *}: \mathrm{D}-\bmod \left(\operatorname{Bun}_{M}\right)_{\mathrm{co}} \rightleftarrows \mathrm{D}-\bmod \left(\operatorname{Bun}_{G}\right)_{\mathrm{co}}: \mathrm{CT}_{\mathrm{co}, ?,}
\end{aligned}
$$

and

$$
\mathrm{CT}_{\mathrm{co}, *}^{\mu}: \mathrm{D}-\bmod \left(\operatorname{Bun}_{G}\right)_{\mathrm{co}} \rightleftarrows \mathrm{D}-\bmod \left(\operatorname{Bun}_{M}^{\mu}\right)_{\mathrm{co}}: \operatorname{Eis}_{\mathrm{co}, ?}^{\mu} .
$$

1.4.6. Finally, from Theorem 1.1.7, we obtain:

Corollary 1.4.7. There are canonical isomorphisms of functors

$$
\operatorname{Eis}_{\mathrm{co}, ?}^{\mu} \simeq \operatorname{Eis}_{\mathrm{co}, *}^{\mu,-}, \quad \operatorname{Eis}_{\mathrm{co}, ?} \simeq \operatorname{Eis}_{\mathrm{co}, *}^{-}
$$

and

$$
\mathrm{CT}_{\mathrm{co}, *}^{\mu} \simeq\left(\mathrm{CT}_{*}^{\mu,-}\right)^{\mathrm{op}} .
$$

To summarize, we also obtain an adjunction

$$
\mathrm{CT}_{\mathrm{co}, *}^{\mu}: \mathrm{D}-\bmod \left(\operatorname{Bun}_{G}\right)_{\mathrm{co}} \rightleftarrows \mathrm{D}-\bmod \left(\operatorname{Bun}_{M}^{\mu}\right)_{\mathrm{co}}: \operatorname{Eis}_{\mathrm{co}, *}^{\mu,-} .
$$

1.4.8. We can ask the following question: does the functor $\mathrm{CT}_{\mathrm{co}, *}^{\mu}$ admit a left adjoint? The answer is "no":

Proof. If $\mathrm{CT}_{\mathrm{co}, *}^{\mu}$ had admitted a left adjoint, by Sect. 1.3.2, the functor $\operatorname{Eis}_{*}^{\mu}$ would have admitted a continuous right adjoint. However, this is not the case, since the functor

$$
\operatorname{Eis}_{*}^{\mu}: \operatorname{D}-\bmod \left(\operatorname{Bun}_{M}^{\mu}\right) \rightarrow \mathrm{D}-\bmod \left(\operatorname{Bun}_{G}\right)
$$

does not preserve compactness.

\subsection{Explicit description of the dual functors.}


1.5.1. For $\left(U \stackrel{j}{\hookrightarrow} \operatorname{Bun}_{G}\right) \in$ op-qc $(G)$ we consider the functor $j^{*}: \mathrm{D}-\bmod \left(\operatorname{Bun}_{G}\right) \rightarrow \mathrm{D}-\bmod (U)$, and its right adjoint $j_{*}$.

Define

$$
j_{\mathrm{co}}^{*}: \mathrm{D}-\bmod \left(\operatorname{Bun}_{G}\right)_{\mathrm{co}} \rightarrow \mathrm{D}-\bmod (U)
$$

as

$$
j_{\mathrm{co}}^{*}:=\left(j_{*}\right)^{\vee}
$$

By Sect. 1.3.2, the functors

$$
j_{\mathrm{co}}^{*}: \mathrm{D}-\bmod \left(\operatorname{Bun}_{G}\right)_{\mathrm{co}} \rightleftarrows \mathrm{D}-\bmod (U): j_{\mathrm{co}, *}
$$

form an adjoint pair, where $j_{\mathrm{co}, *}$ is as in (1.4).

Lemma 1.5.2. The functor $j_{\mathrm{co}, *}$ is fully faithful.

Proof. We need to show that the co-unit of the adjunction

$$
j_{\mathrm{co}}^{*} \circ j_{\mathrm{co}, *} \rightarrow \operatorname{Id}_{\mathrm{D}-\bmod (U)}
$$

is an isomorphism. But this follows from the fact that the corresponding map between the dual functors, i.e.,

$$
j^{*} \circ j_{*} \rightarrow \operatorname{Id}_{\mathrm{D}-\bmod (U)},
$$

is an isomorphism (the latter because $j_{*}: \mathrm{D}-\bmod (U) \rightarrow \mathrm{D}-\bmod \left(\mathrm{Bun}_{G}\right)$ is fully faithful).

1.5.3. By the definition of $\mathrm{D}-\bmod \left(\operatorname{Bun}_{G}\right)_{\mathrm{co}}$, the functor $j_{\mathrm{co}}^{*}$ amounts to a compatible family of functors

$$
j_{\mathrm{co}}^{*} \circ\left(j_{1}\right)_{\mathrm{co}, *}: \mathrm{D}-\bmod \left(U_{1}\right) \rightarrow \mathrm{D}-\bmod (U)
$$

for $\left(U_{1} \stackrel{j_{1}}{\longrightarrow} \operatorname{Bun}_{G}\right) \in$ op-qc $(G)$.

It is easy to see from the definitions that

$$
j_{\mathrm{co}}^{*} \circ\left(j_{1}\right)_{\mathrm{co}, *} \simeq\left(j_{1}^{\prime}\right)_{*} \circ\left(j^{\prime}\right)^{*},
$$

where

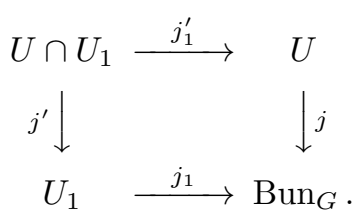

1.5.4. Again, by the definition of the category D-mod( $\left(\operatorname{Bun}_{M}\right)_{\mathrm{co}}$, the functor Eis $\mathrm{co}_{\mathrm{co}, *}$ amounts to a compatible family of functors

$$
\operatorname{Eis}_{\mathrm{co}, *} \circ\left(j_{M}\right)_{\mathrm{co}, *}: \mathrm{D}-\bmod \left(U_{M}\right) \rightarrow \mathrm{D}-\bmod \left(\operatorname{Bun}_{G}\right)_{\mathrm{co}}
$$

for $\left(U_{M} \stackrel{j_{M}}{\longrightarrow} \operatorname{Bun}_{M}\right) \in \operatorname{op}-\mathrm{qc}(M)$.

We now claim:

Proposition 1.5.5. For a given $\left(U_{M} \stackrel{j_{M}}{\longrightarrow} \operatorname{Bun}_{M}\right) \in$ op-qc $(M)$, let $\left(U_{G} \stackrel{j_{G}}{\rightarrow} \operatorname{Bun}_{G}\right) \in$ op-qc $(G)$ be such that

$$
\mathrm{p}\left(\mathrm{q}^{-1}\left(U_{M}\right)\right) \subset U_{G} .
$$

Then there is a canonical isomorphism

$$
\operatorname{Eis}_{\mathrm{co}, *} \circ\left(j_{M}\right)_{\mathrm{co}, *} \simeq\left(j_{G}\right)_{\mathrm{co}, *} \circ\left(j_{G}\right)^{*} \circ \operatorname{Eis}_{*} \circ\left(j_{M}\right)_{*}: \quad \mathrm{D}-\bmod \left(U_{M}\right) \rightarrow \mathrm{D}-\bmod (\operatorname{Bun})_{\mathrm{co}} .
$$


Proof. First, we claim that there is a canonical isomorphism

$$
\operatorname{Eis}_{\mathrm{co}, *} \circ\left(j_{M}\right)_{\mathrm{co}, *} \simeq\left(j_{G}\right)_{\mathrm{co}, *} \circ\left(j_{G}\right)_{\mathrm{co}}^{*} \circ \operatorname{Eis}_{\mathrm{co}, *} \circ\left(j_{M}\right)_{\mathrm{co}, *} .
$$

Indeed, (1.8) follows by passing to dual functors in the isomorphism

$$
\left(j_{M}\right)^{*} \circ \mathrm{CT}_{*} \simeq\left(j_{M}\right)^{*} \circ \mathrm{CT}_{*} \circ\left(j_{G}\right)_{*} \circ\left(j_{G}\right)^{*},
$$

where the latter follows by base change from the definition.

Hence, it remains to establish a canonical isomorphism of functors

$$
\left(j_{G}\right)_{\mathrm{co}}^{*} \circ \operatorname{Eis}_{\mathrm{co}, *} \circ\left(j_{M}\right)_{\mathrm{co}, *} \simeq\left(j_{G}\right)^{*} \circ \operatorname{Eis}_{*} \circ\left(j_{M}\right)_{*}, \quad \mathrm{D}-\bmod \left(U_{M}\right) \rightarrow \mathrm{D}-\bmod \left(U_{G}\right),
$$

i.e., an isomorphism

$$
\left(\left(j_{M}\right)^{*} \circ \mathrm{CT}_{*} \circ\left(j_{G}\right)_{*}\right)^{\vee} \simeq\left(j_{G}\right)^{*} \circ \operatorname{Eis}_{*} \circ\left(j_{M}\right)_{*} .
$$

However, the latter amounts to pull-push along the diagram

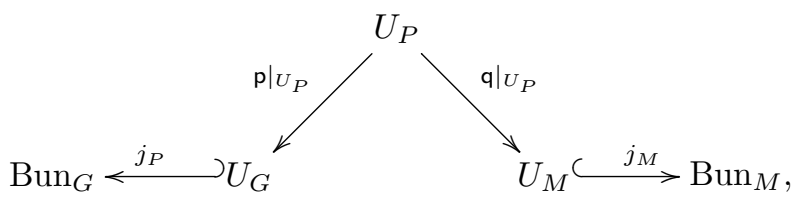

where $U_{P}:=\mathrm{q}^{-1}\left(U_{M}\right)$.

1.5.6. The functor $\mathrm{CT}_{\mathrm{co}, *}$ amounts to a compatible family of functors

$$
\mathrm{CT}_{\mathrm{co}, *} \circ\left(j_{G}\right)_{\mathrm{co}, *}: \mathrm{D}-\bmod \left(U_{G}\right) \rightarrow \mathrm{D}-\bmod \left(\operatorname{Bun}_{M}\right)_{\mathrm{co}}
$$

for $\left(U_{G} \stackrel{j_{G}}{\hookrightarrow} \operatorname{Bun}_{G}\right) \in$ op-qc $(G)$.

In a similar way to Proposition 1.5.5, we have:

Proposition 1.5.7. For a given $\left(U_{G} \stackrel{j_{G}}{\hookrightarrow} \operatorname{Bun}_{G}\right) \in \mathrm{op}-\mathrm{qc}(G)$, let $\left(U_{M} \stackrel{j_{M}}{\longrightarrow} \operatorname{Bun}_{M}\right) \in$ op-qc $(M)$ be such that

Then there is a canonical isomorphism

$$
\mathrm{q}\left(\mathrm{p}^{-1}\left(U_{G}\right)\right) \subset U_{M} .
$$

$$
\mathrm{CT}_{\mathrm{co}, *} \circ\left(j_{G}\right)_{\mathrm{co}, *} \simeq\left(j_{M}\right)_{\mathrm{co}, *} \circ\left(j_{M}\right)^{*} \circ \mathrm{CT}_{*} \circ\left(j_{G}\right)_{*}: \quad \mathrm{D}-\bmod \left(U_{G}\right) \rightarrow \mathrm{D}-\bmod \left(\mathrm{Bun}_{M}\right)_{\mathrm{co}} .
$$

2. Interaction With the NAIVE PSEUdO-IDENTITY AND CUSPIDALITY

\subsection{The naive pseudo-identity functor.}

2.1.1. The following functor

$$
\text { Ps- } \operatorname{Id}_{\mathrm{Bun}_{G}, \text { naive }}: \mathrm{D}-\bmod \left(\operatorname{Bun}_{G}\right)_{\mathrm{co}} \rightarrow \mathrm{D}-\bmod \left(\operatorname{Bun}_{G}\right)
$$

was introduced in [DrGa2, Sect. 4.4.2]:

$$
\begin{aligned}
& \text { For }\left(U_{G} \stackrel{j_{G}}{\hookrightarrow} \operatorname{Bun}_{G}\right) \in \operatorname{op}-q c(G) \text {, the composition } \\
& \qquad \operatorname{Ps}-\operatorname{Id}_{\mathrm{Bun}_{G}, \text { naive }} \circ j_{\mathrm{co}, *}: \operatorname{D}-\bmod \left(U_{G}\right) \rightarrow \mathrm{D} \text {-mod }\left(\operatorname{Bun}_{G}\right)
\end{aligned}
$$

is by definition the functor $j_{*}$.

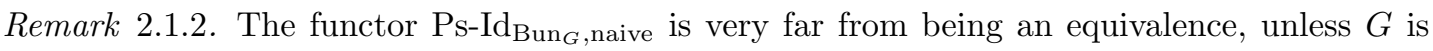
a torus. For example, in [Ga2, Theorem 7.7.2], a particular object of D-mod( $\left.\operatorname{Bun}_{G}\right)_{\text {co }}$ was constructed, which belongs to $\operatorname{ker}\left(\mathrm{Ps}_{\mathrm{S}}-\mathrm{Id}_{\mathrm{Bun}_{G} \text {, naive }}\right)$, as soon as the semi-simple part of $G$ is non-trivial. 
2.1.3. Recall the equivalence:

Funct $_{\text {cont }}\left(\mathrm{D}-\bmod \left(\operatorname{Bun}_{G}\right)_{\mathrm{co}}, \mathrm{D}-\bmod \left(\operatorname{Bun}_{G}\right)\right) \simeq\left(\mathrm{D}-\bmod \left(\operatorname{Bun}_{G}\right)_{\mathrm{co}}\right)^{\vee} \otimes \mathrm{D}-\bmod \left(\operatorname{Bun}_{G}\right) \simeq$ $\simeq \mathrm{D}-\bmod \left(\operatorname{Bun}_{G}\right) \otimes \mathrm{D}-\bmod \left(\operatorname{Bun}_{G}\right) \simeq \mathrm{D}-\bmod \left(\operatorname{Bun}_{G} \times \operatorname{Bun}_{G}\right)$.

According to [DrGa2, Sect. 4.4.3], the functor $\mathrm{Ps}_{\mathrm{s}} \mathrm{Id}_{\mathrm{Bun}_{G}, \text { naive }}$ corresponds to the object

$$
\left(\Delta_{\mathrm{Bun}_{G}}\right)_{*}\left(\omega_{\mathrm{Bun}_{G}}\right) \in \mathrm{D}-\bmod \left(\operatorname{Bun}_{G} \times \operatorname{Bun}_{G}\right),
$$

where $\Delta_{\mathrm{Bun}_{G}}$ denotes the diagonal morphism on $\mathrm{Bun}_{G}$, and $\omega_{y}$ is the dualizing object on a stack $y$ (we take $y=\operatorname{Bun}_{G}$ ).

From here we obtain:

Lemma 2.1.4. There exists a canonical isomorphism $\mathrm{Ps}_{\mathrm{s}}-\mathrm{Id}_{\mathrm{Bun}_{G}, \text { naive }}^{\vee} \simeq \mathrm{Ps}-\mathrm{Id}_{\mathrm{Bun}_{G}, \text { naive }}$.

Proof. This expresses the fact that $\left(\Delta_{\mathrm{Bun}_{G}}\right)_{*}\left(\omega_{\mathrm{Bun}_{G}}\right)$ is equivariant with respect to the flip automorphism of D-mod $\left(\operatorname{Bun}_{G} \times \operatorname{Bun}_{G}\right)$.

Corollary 2.1.5. For $\left(U_{G} \stackrel{j_{G}}{\rightarrow} \operatorname{Bun}_{G}\right) \in$ op-qc $(G)$, we have a canonical isomorphism:

$$
j^{*} \circ \mathrm{Ps}_{\mathrm{S}} \mathrm{Id}_{\mathrm{Bun}_{G}, \text { naive }} \simeq j_{\mathrm{co}}^{*} \text {. }
$$

Proof. Obtained by passing to the dual functors is

$$
\mathrm{Ps}_{-} \mathrm{Id}_{\mathrm{Bun}_{G}, \text { naive }} \circ j_{\mathrm{co}, *} \simeq j_{*} \text {. }
$$

2.1.6. We now claim:

Proposition 2.1.7. There are canonical isomorphisms

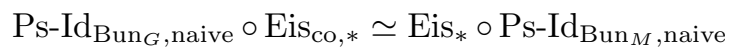

and

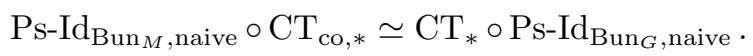

Proof. We will prove the first isomorphism, while the second one is similar.

By definition, we need to construct a compatible family of isomorphisms of functors

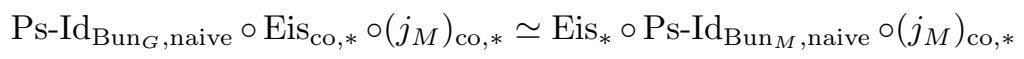

for $\left(U_{M} \stackrel{j_{M}}{\longrightarrow} \operatorname{Bun}_{M}\right) \in$ op-qc $(M)$.

For a given $U_{M}$, let $U_{G}$ be as in Proposition 1.5.5. We rewrite

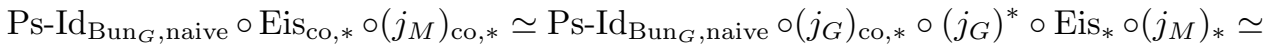

$$
\begin{aligned}
& \simeq\left(j_{G}\right)_{*} \circ\left(j_{G}\right)^{*} \circ \operatorname{Eis}_{*} \circ\left(j_{M}\right)_{*} .
\end{aligned}
$$

However, it is easy to see that for the above choice of $U_{G}$, the natural map

$$
\operatorname{Eis}_{*} \circ\left(j_{M}\right)_{*} \rightarrow\left(j_{G}\right)_{*} \circ\left(j_{G}\right)^{*} \circ \operatorname{Eis}_{*} \circ\left(j_{M}\right)_{*}
$$

is an isomorphism.

Now, by definition,

$$
\operatorname{Eis}_{*} \circ \operatorname{Ps}-\operatorname{Id}_{\mathrm{Bun}_{M}, \text { naive }} \circ\left(j_{M}\right)_{\mathrm{co}, *} \simeq \operatorname{Eis}_{*} \circ\left(j_{M}\right)_{*},
$$

and the assertion follows. (It is clear that these isomorphisms are independent of the choice of $U_{G}$, and hence are compatible under $\left(U_{1}\right)_{M} \hookrightarrow\left(U_{2}\right)_{M}$.) 
D. GAITSGORY

\subsection{Cuspidality.}

2.2.1. Recall that in [DrGa3, Sect. 1.4] the full subcategory

$$
\mathrm{D}-\bmod \left(\operatorname{Bun}_{G}\right)_{\text {cusp }} \subset \mathrm{D}-\bmod \left(\operatorname{Bun}_{G}\right)
$$

was defined as the intersection of the kernels of the functors $\mathrm{CT}_{*}$ for all proper parabolic subgroups $P \subset G$.

Equivalently, let

$$
\mathrm{D}-\bmod \left(\operatorname{Bun}_{G}\right)_{\text {Eis }} \subset \mathrm{D}-\bmod \left(\operatorname{Bun}_{G}\right)
$$

be the full subcategory, generated by the essential images of the functors Eis! for all proper parabolics. From the (Eis!, $\left.\mathrm{CT}_{*}\right)$-adjunction, we obtain

$$
\mathrm{D}-\bmod \left(\operatorname{Bun}_{G}\right)_{\text {cusp }}=\left(\mathrm{D}-\bmod \left(\operatorname{Bun}_{G}\right)_{\text {Eis }}\right)^{\perp} .
$$

2.2.2. We let

$$
\mathrm{D}-\bmod \left(\operatorname{Bun}_{G}\right)_{\mathrm{co}, \text { Eis }} \subset \mathrm{D}-\bmod \left(\operatorname{Bun}_{G}\right)_{\mathrm{co}}
$$

be the full subcategory generated by the essential images of the functors

$$
\operatorname{Eis}_{\mathrm{co}, *}: \mathrm{D}-\bmod \left(\operatorname{Bun}_{M}\right)_{\mathrm{co}} \rightarrow \mathrm{D}-\bmod \left(\operatorname{Bun}_{G}\right)_{\mathrm{co}} .
$$

We define

$$
\mathrm{D}-\bmod \left(\operatorname{Bun}_{G}\right)_{\mathrm{co}, \text { cusp }}:=\left(\mathrm{D}-\bmod \left(\operatorname{Bun}_{G}\right)_{\mathrm{co}, \text { Eis }}\right)^{\perp} .
$$

Equivalently, $\mathrm{D}-\bmod \left(\operatorname{Bun}_{G}\right)_{\mathrm{co}, \text { cusp }}$ is the intersection of the kernels of the functors $\mathrm{CT}_{\mathrm{co}, \text { ? }}$ for all proper parabolics.

\subsubsection{From Corollary 1.4.2 we obtain:}

\section{Corollary 2.2.4.}

(1) An object of $\mathrm{D}-\bmod \left(\mathrm{Bun}_{G}\right)_{c o}$ is cuspidal if and only if its pairing with every object of $\mathrm{D}-\bmod \left(\operatorname{Bun}_{G}\right)_{\text {Eis }}$ is zero under the canonical map

$$
\langle-,-\rangle_{\mathrm{Bun}_{G}}: \mathrm{D}-\bmod \left(\operatorname{Bun}_{G}\right) \times \mathrm{D}-\bmod \left(\operatorname{Bun}_{G}\right)_{\mathrm{co}} \rightarrow \operatorname{Vect}
$$

corresponding to $\mathbf{D}_{\mathrm{Bun}_{G}}$.

(2) The identification $\mathbf{D}_{\mathrm{Bun}_{G}}: \mathrm{D}-\bmod \left(\operatorname{Bun}_{G}\right)^{\vee} \simeq \mathrm{D}-\bmod \left(\operatorname{Bun}_{G}\right)_{\text {co }}$ induces identifications

$$
\left(\mathrm{D}-\bmod \left(\operatorname{Bun}_{G}\right)_{\mathrm{Eis}}\right)^{\vee} \simeq \mathrm{D}-\bmod \left(\operatorname{Bun}_{G}\right)_{\mathrm{co}, \text { Eis }} \text { and }\left(\mathrm{D}-\bmod \left(\operatorname{Bun}_{G}\right)_{\mathrm{cusp}}\right)^{\vee} \simeq \mathrm{D}-\bmod \left(\operatorname{Bun}_{G}\right)_{\mathrm{co}, \text { cusp }} \text {. }
$$

Remark 2.2.5. We will see shortly that $\mathrm{D}-\bmod \left(\mathrm{Bun}_{G}\right)_{\mathrm{co}, \text { cusp }}$ belongs to the intersection of the kernels of the functors $\mathrm{CT}_{\mathrm{co}, *}$ for all proper parabolics. But this inclusion is strict. For example fr $G=S L_{2}$, the object from [Ga2, Theorem 7.7.2] belongs to $\mathrm{CT}_{\mathrm{co}, *}$ (there is only one parabolic to consider), but it does not belong to $\mathrm{D}-\bmod \left(\operatorname{Bun}_{G}\right)_{\mathrm{co}, \mathrm{cusp}}$.

2.2.6. Our goal for the rest of this section is to prove:

Theorem 2.2.7. The restriction of the functor $\mathrm{Ps}_{\mathrm{S}}-\mathrm{Id}_{\mathrm{Bun}_{G}, \text { naive }}$ to

$$
\mathrm{D}-\bmod \left(\operatorname{Bun}_{G}\right)_{\mathrm{co}, \mathrm{cusp}} \subset \mathrm{D}-\bmod \left(\operatorname{Bun}_{G}\right)_{\mathrm{co}}
$$

takes values in $\mathrm{D}-\bmod \left(\operatorname{Bun}_{G}\right)_{\text {cusp }} \subset \mathrm{D}-\bmod \left(\operatorname{Bun}_{G}\right)$, and defines an equivalence

$$
\mathrm{D}-\bmod \left(\operatorname{Bun}_{G}\right)_{\mathrm{co}, \mathrm{cusp}} \rightarrow \mathrm{D}-\bmod \left(\operatorname{Bun}_{G}\right)_{\mathrm{cusp}} \text {. }
$$

\subsection{Support of cuspidal objects.}


2.3.1. The following crucial property of $\mathrm{D}-\bmod \left(\mathrm{Bun}_{G}\right)_{\text {cusp }}$ was established in [DrGa3, Proposition 1.4.6]:

Proposition 2.3.2. There exists an element $\left(\mathcal{U}_{G} \stackrel{J_{G}}{\rightarrow} \operatorname{Bun}_{G}\right) \in$ op-qc $(G)$, such that for any $\mathcal{F} \in \mathrm{D}-\bmod \left(\operatorname{Bun}_{G}\right)_{\text {cusp }}$, the maps

$$
\left(\jmath_{G}\right) ! \circ\left(\jmath_{G}\right)^{*}(\mathcal{F}) \rightarrow \mathcal{F} \rightarrow\left(\jmath_{G}\right)_{*} \circ\left(\jmath_{G}\right)^{*}(\mathcal{F})
$$

are isomorphisms.

2.3.3. We now claim that a parallel phenomenon takes place for $\mathrm{D}-\bmod \left(\operatorname{Bun}_{G}\right)_{\mathrm{co}, \mathrm{cusp}}$ :

Proposition 2.3.4. For any $\mathcal{F} \in \mathrm{D}-\bmod \left(\operatorname{Bun}_{G}\right)_{\mathrm{co}, \text { cusp }}$, the map

$$
\mathcal{F} \rightarrow\left(\jmath_{G}\right)_{\mathrm{co}, *} \circ\left(\jmath_{G}\right)_{\mathrm{co}}^{*}(\mathcal{F})
$$

is an isomorphism.

Proof. We need to show that the map from the tautological embedding

$$
\mathrm{D}-\bmod \left(\operatorname{Bun}_{G}\right)_{\mathrm{co}, \text { cusp }} \stackrel{\mathbf{e}_{\mathrm{co}}}{\longrightarrow} \mathrm{D}-\bmod \left(\operatorname{Bun}_{G}\right)_{\mathrm{co}}
$$

to the composition

$$
\mathrm{D}-\bmod \left(\operatorname{Bun}_{G}\right)_{\mathrm{co}, \text { cusp }} \stackrel{\mathbf{e}_{\mathrm{co}}}{\longrightarrow} \mathrm{D}-\bmod \left(\operatorname{Bun}_{G}\right)_{\mathrm{co}} \stackrel{\left(\jmath_{G}\right)_{\mathrm{oo}}^{*}}{\longrightarrow} \mathrm{D}-\bmod \left(\mathcal{U}_{G}\right) \stackrel{\left(\jmath_{G}\right)_{\mathrm{co}, *}}{\longrightarrow} \mathrm{D}-\bmod \left(\operatorname{Bun}_{G}\right)_{\mathrm{co}}
$$

is an isomorphism.

Note that in terms of the identification of Corollary 2.2.4(b), the dual of the embedding $\mathbf{e}_{\mathrm{co}}$ of $(2.1)$ is the functor

$$
\text { f : } \mathrm{D}-\bmod \left(\operatorname{Bun}_{G}\right) \rightarrow \mathrm{D}-\bmod \left(\operatorname{Bun}_{G}\right)_{\mathrm{cusp}},
$$

left adjoint to the tautological embedding $\mathrm{D}-\bmod \left(\operatorname{Bun}_{G}\right)_{\text {cusp }} \stackrel{\mathrm{e}}{\hookrightarrow} \mathrm{D}-\bmod \left(\operatorname{Bun}_{G}\right)$.

Hence, by duality, we need to show that the functor (2.2) maps isomorphically to the composition

$$
\mathrm{D}-\bmod \left(\operatorname{Bun}_{G}\right) \stackrel{\left(\jmath_{G}\right)^{*}}{\longrightarrow} \mathrm{D}-\bmod \left(\mathcal{U}_{G}\right) \stackrel{\left(\jmath_{G}\right)_{*}^{*}}{\longrightarrow} \mathrm{D}-\bmod \left(\operatorname{Bun}_{G}\right) \stackrel{\mathbf{f}}{\longrightarrow} \mathrm{D}-\bmod \left(\operatorname{Bun}_{G}\right)_{\mathrm{cusp}} .
$$

The latter is equivalent to the fact that any $\mathcal{F}^{\prime} \in \mathrm{D}-\bmod \left(\operatorname{Bun}_{G}\right)$ for which $\jmath_{G}^{*}\left(\mathcal{F}^{\prime}\right)=0$, is left-orthogonal to D-mod $\left(\operatorname{Bun}_{G}\right)_{\text {cusp. }}$. However, this follows from the isomorphism

$$
\mathcal{F} \rightarrow\left(\jmath_{G}\right)_{*} \circ\left(\jmath_{G}\right)^{*}(\mathcal{F}), \quad \mathcal{F} \in \mathrm{D}-\bmod \left(\operatorname{Bun}_{G}\right)_{\text {cusp }}
$$

of Proposition 2.3.2.

\subsection{Description of the cuspidal category.}

2.4.1. We claim:

Proposition 2.4.2. Let $\mathcal{F} \in \mathrm{D}-\bmod \left(\operatorname{Bun}_{G}\right)_{\text {co }}$ be such that there exists $\left(U \stackrel{j}{\hookrightarrow} \operatorname{Bun}_{G}\right) \in \mathrm{op}-\mathrm{qc}(G)$ such that the map

$$
\mathcal{F} \rightarrow j_{\mathrm{co}, *} \circ j_{\mathrm{co}}^{*}(\mathcal{F})
$$

is an isomorphism. Then $\mathcal{F} \in \mathrm{D}-\bmod \left(\operatorname{Bun}_{G}\right)_{\mathrm{co}, \mathrm{cusp}}$ if and only if $\mathrm{CT}_{\mathrm{co}, *}(\mathcal{F})=0$ for all proper parabolics. 
Proof. Recall that

$$
\langle-,-\rangle_{\mathrm{Bun}_{G}}: \mathrm{D}-\bmod \left(\operatorname{Bun}_{G}\right)_{\mathrm{co}} \times \mathrm{D}-\bmod \left(\mathrm{Bun}_{G}\right) \rightarrow \text { Vect }
$$

denotes the pairing corresponding to the identification

$$
\mathbf{D}_{\mathrm{Bun}_{G}}: \mathrm{D}-\bmod \left(\operatorname{Bun}_{G}\right)^{\vee} \simeq \mathrm{D}-\bmod \left(\operatorname{Bun}_{G}\right)_{\mathrm{co}} .
$$

On the one hand, by Corollary 1.4.2, for $\mathcal{F}_{G} \in \mathrm{D}-\bmod \left(\operatorname{Bun}_{G}\right)_{\text {co }}$, the condition that $\mathcal{F}_{G}$ be right-orthogonal to the essential image of $\operatorname{Eis}_{\mathrm{co}_{*} *}$ for a given parabolic $P$ is equivalent to

$$
\left\langle\operatorname{Eis!}_{!}\left(\mathcal{F}_{M}\right), \mathcal{F}_{G}\right\rangle_{\text {Bun }_{G}}=0, \quad \mathcal{F}_{M} \in \mathrm{D}-\bmod \left(\operatorname{Bun}_{M}\right) .
$$

If $\mathcal{F}_{G}=j_{\mathrm{co}, *}\left(\mathcal{F}_{U}\right)$, then the above is equivalent to

$$
\left\langle j^{*} \circ \operatorname{Eis!}\left(\mathcal{F}_{M}\right), \mathcal{F}_{U}\right\rangle_{U}=0,
$$

where

$$
\langle-,-\rangle_{U}: \mathrm{D}-\bmod (U)_{\mathrm{co}} \times \mathrm{D}-\bmod (U) \rightarrow \operatorname{Vect}
$$

is the pairing corresponding to $\mathbf{D}_{U}: \mathrm{D}-\bmod (U)^{\vee} \simeq \mathrm{D}-\bmod (U)$. to

On the other hand, the condition that $\mathrm{CT}_{\mathrm{co}, *}\left(\mathcal{F}_{G}\right)=0$ for the same parabolic is equivalent i.e.,

$$
\left\langle\operatorname{Eis}_{*}\left(\mathcal{F}_{M}\right), \mathcal{F}_{G}\right\rangle_{\mathrm{Bun}_{G}},
$$$$
\left\langle j^{*} \circ \operatorname{Eis}_{*}\left(\mathcal{F}_{M}\right), \mathcal{F}_{U}\right\rangle_{U}=0 .
$$

Hence, the assertion of Proposition 2.4.2 follows from the next one, proved in Sect. 2.5:

Proposition 2.4.3.

(a) For $\mathcal{F}_{M} \in \mathrm{D}-\bmod \left(\operatorname{Bun}_{M}\right)$, the object $\operatorname{Eis}_{*}\left(\mathcal{F}_{M}\right)$ admits an increasing filtration (indexed by a poset) with subquotients of the form $\operatorname{Eis}_{!}\left(\mathcal{F}_{M}^{\alpha}\right), \mathcal{F}_{M}^{\alpha} \in \mathrm{D}-\bmod \left(\operatorname{Bun}_{M}\right)$.

(b) Assume that $\mathcal{F}_{M}$ is supported on finitely many connected components of $\operatorname{Bun}_{M}$, and let $\left(U \stackrel{j}{\hookrightarrow} \operatorname{Bun}_{G}\right) \in \operatorname{op}-\mathrm{qc}(G)$. Then:

(i) The objects $j^{*} \circ \operatorname{Eis}_{!}\left(\mathcal{F}_{M}^{\alpha}\right)$ from point (a) are zero for all but finitely many $\alpha$ 's.

(ii) The object $j^{*} \circ \operatorname{Eis!}_{!}\left(\mathcal{F}_{M}\right)$ is a finite successive extension of objects of the form $j^{*} \circ \operatorname{Eis}_{*}\left(\mathcal{F}_{M}^{\alpha}\right)$, $\mathcal{F}_{M}^{\alpha} \in \mathrm{D}-\bmod \left(\operatorname{Bun}_{M}\right)$.

2.4.4. We now observe:

Proposition 2.4.5. Let $\mathcal{F} \in \mathrm{D}-\bmod \left(\mathrm{Bun}_{G}\right)_{\text {co }}$ be such that there exists $\left(U_{G} \stackrel{j_{G}}{\hookrightarrow} \operatorname{Bun}_{G}\right) \in$ op-qc $(G)$ such that the map

$$
\mathcal{F} \rightarrow\left(j_{G}\right)_{\mathrm{co}, *} \circ\left(j_{G}\right)_{\mathrm{co}}^{*}(\mathcal{F})
$$

is an isomorphism. Then $\mathrm{Ps}_{-}-\mathrm{Id}_{\mathrm{Bun}_{G}, \text { naive }}(\mathcal{F}) \in \mathrm{D}-\bmod \left(\mathrm{Bun}_{G}\right)_{\text {cusp }}$ if and only if $\mathrm{CT}_{\mathrm{co}, *}(\mathcal{F})=0$ for all proper parabolics.

Proof. We claim that for $\mathcal{F}$ satisfying the condition of the proposition, for a given parabolic $P$,

$$
\mathrm{CT}_{*} \circ \operatorname{Ps}_{-\mathrm{Id}_{\text {Bun }}, \text { naive }}(\mathcal{F})=0 \Leftrightarrow \mathrm{CT}_{\mathrm{co}, *}(\mathcal{F})=0 .
$$

Indeed, the implication $\Leftarrow$ holds for any $\mathcal{F}$ by Proposition 2.1.7.

Conversely, let $\left(U_{M} \stackrel{j_{M}}{\longrightarrow} \operatorname{Bun}_{M}\right) \in$ op-qc $(M)$ be as in Proposition 1.5.7. For

$$
\mathcal{F} \simeq\left(j_{G}\right)_{\mathrm{co}, *}\left(\mathcal{F}_{U_{G}}\right),
$$


by Proposition 1.5.7, we have

$$
\begin{aligned}
\mathrm{CT}_{\mathrm{co}, *}(\mathcal{F}) \simeq\left(j_{M}\right)_{\mathrm{co}, *} \circ\left(j_{M}\right)^{*} \circ \mathrm{CT}_{*} \circ\left(j_{G}\right)_{*}\left(\mathcal{F}_{U_{G}}\right) \simeq \\
\simeq\left(j_{M}\right)_{\mathrm{co}, *} \circ\left(j_{M}\right)^{*} \circ \mathrm{CT}_{*} \circ \mathrm{Ps}_{-} \mathrm{Id}_{\mathrm{Bun}_{G}, \text { naive }} \circ\left(j_{G}\right)_{\mathrm{co}, *}\left(\mathcal{F}_{U_{G}}\right) \simeq \\
\simeq\left(j_{M}\right)_{\mathrm{co}, *} \circ\left(j_{M}\right)^{*} \circ \mathrm{CT}_{*} \circ \operatorname{Ps}_{-\mathrm{Id}_{\text {Bun }}, \text { naive }}(\mathcal{F}) .
\end{aligned}
$$

2.4.6. Combining Propositions 2.3.4, 2.4.2 and 2.4.5 we obtain:

Corollary 2.4.7. For $\mathcal{F} \in \mathrm{D}-\bmod \left(\operatorname{Bun}_{G}\right)_{\text {co }}$ the following conditions are equivalent:

(i) $\mathcal{F} \in \mathrm{D}-\bmod \left(\operatorname{Bun}_{G}\right)_{\text {co,cusp }}$;

(ii) There exists $\left(U \stackrel{j}{\hookrightarrow} \operatorname{Bun}_{G}\right) \in \operatorname{op}-\mathrm{qc}(G)$ such that the map $\mathcal{F} \rightarrow j_{\mathrm{co}, *} \circ j_{\mathrm{co}}^{*}(\mathcal{F})$ is an isomorphism and $\mathrm{Ps}_{-}-\mathrm{Id}_{\mathrm{Bun}_{G}, \text { naive }}(\mathcal{F}) \in \mathrm{D}-\bmod \left(\operatorname{Bun}_{G}\right)_{\text {cusp }}$.

(ii') There exists $\left(U \stackrel{j}{\hookrightarrow} \operatorname{Bun}_{G}\right) \in$ op-qc $(G)$ such that the map $\mathcal{F} \rightarrow j_{\mathrm{co}, *} \circ j_{\mathrm{co}}^{*}(\mathcal{F})$ is an isomorphism and $\mathrm{CT}_{\mathrm{co}, *}(\mathcal{F})=0$ for all proper parabolics.

(iii) For $\left(\mathcal{U}_{G} \stackrel{\jmath_{G}}{\hookrightarrow} \operatorname{Bun}_{G}\right) \in \mathrm{op}-\mathrm{qc}(G)$ as in Proposition 2.3.2, the map $\mathcal{F} \rightarrow\left(\jmath_{G}\right)_{\mathrm{co}, *} \circ\left(\jmath_{G}\right)_{\mathrm{co}}^{*}(\mathcal{F})$ is an isomorphism and $\mathrm{Ps}_{-}-\mathrm{Id}_{\mathrm{Bun}_{G}, \text { naive }}(\mathcal{F}) \in \mathrm{D}-\bmod \left(\operatorname{Bun}_{G}\right)_{\text {cusp }}$.

(iii') For $\left(\mathcal{U}_{G} \stackrel{J_{G}}{\hookrightarrow} \operatorname{Bun}_{G}\right) \in \mathrm{op}-\mathrm{qc}(G)$ as in Proposition 2.3.2, the map $\mathcal{F} \rightarrow\left(\jmath_{G}\right)_{\mathrm{co}, *} \circ\left(\jmath_{G}\right)_{\mathrm{co}}^{*}(\mathcal{F})$ is an isomorphism and $\mathrm{CT}_{\mathrm{co}, *}(\mathcal{F})=0$ for all proper parabolics.

2.4.8. Proof of Theorem 2.2.7. From Corollary 2.4.7 we obtain that the functor Ps- $\operatorname{Id}_{\mathrm{Bun}_{G} \text {, naive }}$ sends

$$
\mathrm{D}-\bmod \left(\operatorname{Bun}_{G}\right)_{\text {co,cusp }} \rightarrow \mathrm{D}-\bmod \left(\operatorname{Bun}_{G}\right)_{\text {cusp }} .
$$

We construct the inverse functor as follows. Let $\left(\mathcal{U}_{G} \stackrel{J_{G}}{\rightarrow} \operatorname{Bun}_{G}\right) \in$ op-qc $(G)$ be as in Proposition 2.3.2. The sought-for functor

$$
\mathrm{D}-\bmod \left(\operatorname{Bun}_{G}\right)_{\text {cusp }} \rightarrow \mathrm{D}-\bmod \left(\operatorname{Bun}_{G}\right)_{\mathrm{co}}
$$

is

$$
\mathcal{F} \mapsto\left(\jmath_{G}\right)_{\mathrm{co}, *} \circ\left(\jmath_{G}\right)^{*}(\mathcal{F}) .
$$

We claim that the image of this functor lands in $\mathrm{D}-\bmod \left(\operatorname{Bun}_{G}\right)_{\mathrm{co}, \mathrm{cusp}}$. Indeed, by Proposition 2.4.5, its suffices to check that

$$
\text { Ps- } \operatorname{Id}_{\mathrm{Bun}_{G}, \text { naive }} \circ\left(\jmath_{G}\right)_{\mathrm{co}, *} \circ\left(\jmath_{G}\right)^{*}(\mathcal{F}) \in \mathrm{D}-\bmod \left(\operatorname{Bun}_{G}\right)_{\text {cusp }} \text {. }
$$

However,

$$
\text { Ps-Id }_{\mathrm{Bun}_{G}, \text { naive }} \circ\left(\jmath_{G}\right)_{\mathrm{co}, *} \circ\left(\jmath_{G}\right)^{*}(\mathcal{F}) \simeq\left(\jmath_{G}\right)_{*} \circ\left(\jmath_{G}\right)^{*}(\mathcal{F}),
$$

and the latter is isomorphic to $\mathcal{F}$ by Proposition 2.4.5.

Let us now check that the two functors are inverses of each other. However, we have just shown that the composition

$$
\mathrm{D}-\bmod \left(\operatorname{Bun}_{G}\right)_{\text {cusp }} \rightarrow \mathrm{D}-\bmod \left(\operatorname{Bun}_{G}\right)_{\text {co,cusp }} \rightarrow \mathrm{D}-\bmod \left(\operatorname{Bun}_{G}\right)_{\text {cusp }}
$$

is isomorphic to the identity functor.

For the composition in the other direction, for $\mathcal{F} \in \mathrm{D}-\bmod \left(\mathrm{Bun}_{G}\right)_{\text {co,cusp }}$ we consider

$$
\left(\jmath_{G}\right)_{\mathrm{co}, *} \circ\left(\jmath_{G}\right)^{*} \circ \mathrm{Ps}-\operatorname{Id}_{\mathrm{Bun}_{G}, \text { naive }}(\mathcal{F}),
$$

which by Corollary 2.1.5 is isomorphic to

$$
\left(\jmath_{G}\right)_{\mathrm{co}, *} \circ\left(\jmath_{G}\right)_{\mathrm{co}}^{*}(\mathcal{F}),
$$


and the latter is isomorphic to $\mathcal{F}$ by Proposition 2.3.4.

\subsection{Proof of Proposition 2.4.3.}

2.5.1. The proof of the proposition uses the relative compactification $\operatorname{Bun}_{P} \stackrel{r}{\hookrightarrow} \widetilde{\operatorname{Bun}}_{P}$ of the map p, introduced in [BG, Sect. 1.3.6]:

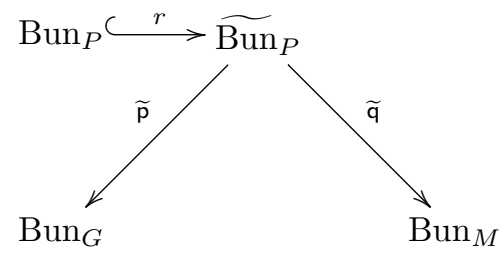

Note that for $\mathcal{F}_{M} \in \mathrm{D}$-mod $\left(\operatorname{Bun}_{M}\right)$, we have

$$
\operatorname{Eis}_{*}\left(\mathcal{F}_{M}\right) \simeq \widetilde{\mathrm{p}}_{*}\left(\widetilde{\mathrm{q}}^{!}\left(\mathcal{F}_{M}\right) \stackrel{!}{\otimes} r_{*}\left(\omega_{\mathrm{Bun}_{P}}\right)\right) \simeq \widetilde{\mathrm{p}}_{!}\left(\widetilde{\mathrm{q}}^{!}\left(\mathcal{F}_{M}\right) \stackrel{!}{\otimes} r_{*}\left(\omega_{\mathrm{Bun}_{P}}\right)\right),
$$

the latter isomorphism due to the fact that $\widetilde{\mathrm{p}}$ is proper. Here the notation $\stackrel{!}{\otimes}$ (and, in the sequel, $\stackrel{*}{\otimes)}$ follows [DrGa3, Sect. 1.1.5].

Recall now that according to [BG, Theorem 5.1.5], the object

$$
r_{*}\left(\omega_{\mathrm{Bun}_{P}}\right) \in \mathrm{D}-\bmod \left(\widetilde{\operatorname{Bun}_{P}}\right)
$$

is universally locally acyclic (a.k.a. ULA) ${ }^{4}$ with respect to the map $\widetilde{\mathrm{q}}$. This implies that

$$
\widetilde{\mathrm{q}}^{!}\left(\mathcal{F}_{M}\right) \stackrel{!}{\otimes} r_{*}\left(\omega_{\mathrm{Bun}_{P}}\right) \simeq \widetilde{\mathrm{q}}^{*}\left(\mathcal{F}_{M}\right) \stackrel{*}{\otimes} r_{*}\left(\omega_{\mathrm{Bun}_{P}}\right)\left[-2 \operatorname{dim}\left(\operatorname{Bun}_{M}\right)\right] .
$$

Thus, we obtain that, up to a cohomological shift, $\operatorname{Eis}_{*}\left(\mathcal{F}_{M}\right)$ is isomorphic to

$$
\widetilde{\mathrm{p}}_{!}\left(\widetilde{\mathrm{q}}^{*}\left(\mathcal{F}_{M}\right) \stackrel{*}{\otimes} r_{*}\left(\omega_{\mathrm{Bun}_{P}}\right)\right) .
$$

2.5.2. Let $\Lambda_{G, P}^{\text {pos }}$ be the monoid of linear combinations

$$
\theta=\sum_{i} n_{i} \cdot \alpha_{i}
$$

where $n_{i} \in \mathbb{Z}^{\geq 0}$ and $\alpha_{i}$ is a simple coroot of $G$, which is not in $M$.

For each $\theta$, we let $\operatorname{Mod}_{\mathrm{Bun}_{M}}^{\theta,+}$ be a version of the Hecke stack, introduced in [BFGM, Sect. $3.1]$ :

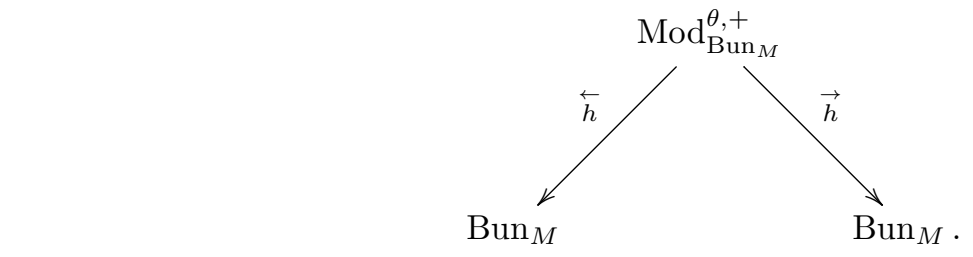

Set

$$
\operatorname{Mod}_{\mathrm{Bun}_{P}}^{\theta,+}:=\operatorname{Bun}_{P} \underset{\operatorname{Bun}_{M}}{\times} \operatorname{Mod}_{\operatorname{Bun}_{M}}^{\theta,+},
$$

${ }^{4}$ See [DrGa3, Sect. 1.1.5] for what the ULA condition means. 
where the fiber product is formed using the map $\overleftarrow{h}: \operatorname{Mod}_{\operatorname{Bun}_{M}}^{\theta,+} \rightarrow \operatorname{Bun}_{M}$

According to [BG, Proposition 6.2.5], there is a canonically defined locally closed embedding

$$
r^{\theta}: \operatorname{Mod}_{\operatorname{Bun}_{P}}^{\theta,+} \rightarrow \widetilde{\operatorname{Bun}}_{P}
$$

making the following diagram commute

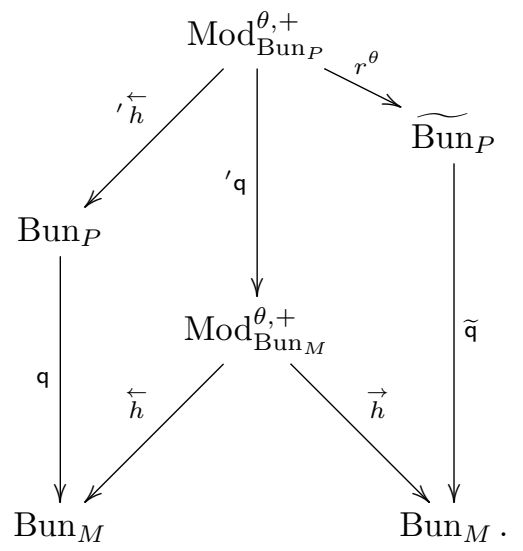

(The right diamond is intentionally lopsided to emphasize that it is not Cartesian.)

Furthermore,

$$
\widetilde{\operatorname{Bun}}_{P}=\bigsqcup_{\theta \in \Lambda_{G, P}^{\text {pos }}} r^{\theta}\left(\operatorname{Mod}_{\operatorname{Bun}_{P}}^{\theta,+}\right)
$$

For $\theta=0$, the map $\overleftarrow{h}$ is an isomorphism, and the resulting map

$$
\operatorname{Bun}_{P} \simeq \operatorname{Mod}_{\operatorname{Bun}_{P}}^{0,+} \stackrel{r^{0}}{\hookrightarrow} \widetilde{\operatorname{Bun}}_{P}
$$

is the map $r$ in (2.3).

The following is easy to see from the construction:

Lemma 2.5.3. For $\left(U \stackrel{j}{\hookrightarrow} \operatorname{Bun}_{G}\right) \in$ op-qc $(G)$ and $\mu \in \pi_{1}(M)$, the preimage of $U \times \operatorname{Bun}_{M}^{\mu}$ under the map

$$
\operatorname{Mod}_{\mathrm{Bun}_{P}}^{\theta,+} \stackrel{r^{\theta}}{\stackrel{\operatorname{Bun}_{P}}{\widetilde{\mathrm{p}} \times \widetilde{\mathrm{q}}} \longrightarrow} \operatorname{Bun}_{G} \times \operatorname{Bun}_{M}
$$

is empty for all but finitely many elements $\theta$.

2.5.4. The decomposition (2.5) endows the object

$$
r_{*}\left(\omega_{\mathrm{Bun}_{P}}\right) \in \mathrm{D}-\bmod \left(\widetilde{\operatorname{Bun}}_{P}\right)
$$

with an increasing filtration, indexed by the poset $\Lambda_{G, P}^{\text {pos }}$, with the $\theta$ subquotient equal to

$$
\left(r^{\theta}\right) ! \circ\left(r^{\theta}\right)^{*} \circ r_{*}\left(\omega_{\mathrm{Bun}_{P}}\right) .
$$

Hence, by the projection formula, the object in (2.4) admits a filtration, indexed by $\Lambda_{G, P}^{\text {pos }}$, with the $\theta$ subquotient equal to

$$
\widetilde{\mathrm{p}}_{!} \circ r_{!}^{\theta}\left(\left(r^{\theta}\right)^{*} \circ \widetilde{\mathrm{q}}^{*}\left(\mathcal{F}_{M}\right) \stackrel{*}{\otimes}\left(r^{\theta}\right)^{*} \circ r_{*}\left(\omega_{\mathrm{Bun}_{P}}\right)\right) .
$$

Moreover, if $\mathcal{F}_{M}$ is supported on finitely many components of $\operatorname{Bun}_{M}$, the restriction of the subquotient (2.6) to $U \in$ op-qc $(G)$ is zero for all but finitely many $\theta$ by Lemma 2.5.3. 
2.5.5. We have the following assertion, proved by the same argument as [BG, Theorem 6.2.10]:

Lemma 2.5.6. The object $\left(r^{\theta}\right)^{*} \circ r_{*}\left(\omega_{\mathrm{Bun}_{P}}\right) \in \mathrm{D}-\bmod \left(\operatorname{Mod}_{\mathrm{Bun}_{P}}^{\theta,+}\right)$ is lisse when !-restricted to the fiber of the map

$$
\text { 'q : } \operatorname{Mod}_{\mathrm{Bun}_{P}}^{\theta,+} \rightarrow \operatorname{Mod}_{\mathrm{Bun}_{M}}^{\theta,+}
$$

over any k-point of $\operatorname{Mod}_{\mathrm{Bun}_{M}}^{\theta,+}$.

Corollary 2.5.7. $\left(r^{\theta}\right)^{*} \circ r_{*}\left(\omega_{\mathrm{Bun}_{P}}\right) \simeq^{\prime} \mathrm{q}^{*}\left(\mathcal{K}^{\theta}\right)$ for some $\mathcal{K}^{\theta} \in \mathrm{D}-\bmod \left(\operatorname{Mod}_{\operatorname{Bun}_{M}}^{\theta,+}\right)$.

Proof. Follows from Lemma 2.5.6 plus the combination of the following three facts: (1) the map 'q is smooth; (2) $\left(r^{\theta}\right)^{*} \circ r_{*}\left(\omega_{\mathrm{Bun}_{P}}\right)$ is holonomic with regular singularities; (3) the fibers of the map 'q are contractible (and hence any RS local system on such a fiber is canonically trivial).

2.5.8. By Corollary 2.5.7, we can rewrite the subquotient (2.6) as

$$
\widetilde{\mathrm{p}}_{!} \circ r_{!}^{\theta}\left(\left(r^{\theta}\right)^{*} \circ \widetilde{\mathrm{q}}^{*}\left(\mathcal{F}_{M}\right) \stackrel{*}{\otimes}\left({ }^{\prime} \mathrm{q}\right)^{*}\left(\mathcal{K}^{\theta}\right)\right),
$$

and further, using the fact that

$$
\widetilde{\mathrm{q}} \circ r^{\theta}=\vec{h} \circ^{\prime} \mathrm{q} \text { and } \widetilde{\mathrm{p}} \circ r^{\theta}=\mathrm{p} \circ{ }^{\prime} \overleftarrow{h}
$$

as

$$
\mathrm{p}_{!} \circ \overleftarrow{h}^{h} ! \circ^{\prime} \mathrm{q}^{*}\left(\vec{h}^{*}\left(\mathcal{F}_{M}\right) \stackrel{*}{\otimes} \mathcal{K}^{\theta}\right) \simeq \mathrm{p} ! \circ \mathrm{q}^{*}\left(\overleftarrow{h}_{!}\left(\vec{h}^{*}\left(\mathcal{F}_{M}\right) \stackrel{*}{\otimes} \mathcal{K}^{\theta}\right)\right)
$$

To summarize, we identify the subquotient (2.6) with

$$
\text { Eis! }\left(\overleftarrow{h}_{!}\left(\vec{h}^{*}\left(\mathcal{F}_{M}\right) \stackrel{*}{\otimes} \mathcal{K}^{\theta}\right)\right)
$$

as required in Proposition 2.4.3(a). The finiteness assertion in Proposition 2.4.3(b)(i) follows from the finiteness at the end of Sect. 2.5.4.

2.5.9. The proof of Proposition 2.4.3(b)(ii) is similar, but with the following modification:

Let $k_{\mathrm{Bun}_{P}} \in \mathrm{D}-\bmod \left(\mathrm{Bun}_{P}\right)$ be the "constant sheaf" D-module, i.e., the Verdier dual of $\omega_{\mathrm{Bun}_{P}}$.

Then the object

$$
r_{!}\left(k_{\mathrm{Bun}_{P}}\right) \in \mathrm{D}-\bmod \left(\widetilde{\operatorname{Bun}_{P}}\right)
$$

admits a decreasing filtration, indexed by the poset $\Lambda_{G, P}^{\mathrm{pos}}$, with the $\theta$ subquotient being

$$
\left(r^{\theta}\right)_{*} \circ\left(r^{\theta}\right)^{!} \circ r_{!}\left(k_{\mathrm{Bun}_{P}}\right) .
$$

However, this filtration is finite on the preimage of $U \times \operatorname{Bun}_{M}^{\mu}$ for any $U \in$ op-qc $(G)$ and $\mu \in \pi_{1}(M)$ under the map $\widetilde{\mathbf{p}} \times \widetilde{\mathrm{q}}$, again by Lemma 2.5 .3 .

\section{INTERACTION WITH THE GENUINE PSEUDO-IDENTITY FUNCTOR}

\subsection{The pseudo-identity functor.}


3.1.1. We now recall that in [DrGa2, Sect. 4.4.8] another functor, denoted

$$
\mathrm{Ps}_{\mathrm{s}} \mathrm{Id}_{\mathrm{Bun}_{G}, !}: \mathrm{D}-\bmod \left(\operatorname{Bun}_{G}\right)_{\mathrm{co}} \rightarrow \mathrm{D}-\bmod \left(\operatorname{Bun}_{G}\right)
$$

was introduced.

Namely, in terms of the equivalences

$$
\begin{array}{r}
\text { Funct }_{\text {cont }}\left(\mathrm{D}-\bmod \left(\mathrm{Bun}_{G}\right)_{\mathrm{co}}, \mathrm{D}-\bmod \left(\operatorname{Bun}_{G}\right)\right) \simeq\left(\mathrm{D}-\bmod \left(\mathrm{Bun}_{G}\right)_{\mathrm{co}}\right)^{\vee} \otimes \mathrm{D}-\bmod \left(\operatorname{Bun}_{G}\right) \simeq \\
\simeq \mathrm{D}-\bmod \left(\operatorname{Bun}_{G}\right) \otimes \mathrm{D}-\bmod \left(\operatorname{Bun}_{G}\right) \simeq \mathrm{D}-\bmod \left(\operatorname{Bun}_{G} \times \operatorname{Bun}_{G}\right),
\end{array}
$$

the functor Ps-Id $\mathrm{Bun}_{G}$, corresponds to the object

$$
\left(\Delta_{\mathrm{Bun}_{G}}\right) !\left(k_{\mathrm{Bun}_{G}}\right) \in \mathrm{D}-\bmod \left(\operatorname{Bun}_{G} \times \operatorname{Bun}_{G}\right) .
$$

3.1.2. Note the following feature of the functor Ps- $\mathrm{Id}_{\mathrm{Bun}_{G}, !}$, parallel to one for Ps- $\mathrm{Id}_{\mathrm{Bun}_{G}, \text { naive }}$, given by Lemma 2.1.4.

Lemma 3.1.3. Under the identification $\mathrm{D}-\bmod \left(\operatorname{Bun}_{G}\right)^{\vee} \simeq \mathrm{D}-\bmod \left(\mathrm{Bun}_{G}\right)_{\mathrm{co}}$, we have

$$
\left(\mathrm{Ps}_{-}-\mathrm{Id}_{\mathrm{Bun}_{G}, !}\right)^{\vee} \simeq \mathrm{Ps}_{\mathrm{s}}-\mathrm{Id}_{\mathrm{Bun}_{G}, !} \cdot
$$

Proof. This is just the fact that the object $\left(\Delta_{\mathrm{Bun}_{G}}\right) !\left(k_{\mathrm{Bun}_{G}}\right) \in \mathrm{D}-\bmod \left(\operatorname{Bun}_{G} \times \operatorname{Bun}_{G}\right)$ is equivariant with respect to the flip.

3.1.4. The goal of this section and the next is to prove:

Theorem 3.1.5. The functor $\mathrm{Ps}_{-} \mathrm{Id}_{\mathrm{Bun}_{G}, !}$ is an equivalence.

The proof will rely on a certain geometric result, namely, Proposition 3.2.6 which will be proved in $[\mathrm{Sch}]$.

\subsection{Relation between the two functors.}

3.2.1. Consider again the map

$$
\Delta_{\mathrm{Bun}_{G}}: \operatorname{Bun}_{G} \rightarrow \operatorname{Bun}_{G} \times \operatorname{Bun}_{G} .
$$

It naturally factors as

$$
\operatorname{Bun}_{G} \stackrel{\operatorname{id}_{\mathrm{Bun}_{G}}^{Z}}{\rightarrow} \operatorname{Bun}_{G} \times B\left(Z_{G}\right) \stackrel{\Delta_{\mathrm{Bun}_{G}}^{Z}}{\longrightarrow} \operatorname{Bun}_{G} \times \operatorname{Bun}_{G},
$$

where:

- $Z_{G}$ denotes the center of $G$, and $B\left(Z_{G}\right)$ is its classifying stack;

- The map id $\mathrm{Bun}_{G}^{Z}$ is given by the identity map $\operatorname{Bun}_{G} \rightarrow \operatorname{Bun}_{G}$, and

$$
\operatorname{Bun}_{G} \rightarrow \mathrm{pt} \stackrel{\text { triv }}{\rightarrow} B\left(Z_{G}\right),
$$

where triv : pt $\rightarrow B\left(Z_{G}\right)$ corresponds to the trivial $Z_{G}$-bundle;

- The composition $\operatorname{pr}_{1} \circ \Delta_{\mathrm{Bun}_{G}}^{Z}$ is projection on the first factor $\operatorname{Bun}_{G} \times B\left(Z_{G}\right) \rightarrow \operatorname{Bun}_{G}$;

- The composition $\operatorname{pr}_{2} \circ \Delta_{\mathrm{Bun}_{G}}^{Z}$ is given by the natural action of $B\left(Z_{G}\right)$ on $\mathrm{Bun}_{G}$.

Remark 3.2.2. Note that if $G$ is a torus, the map $\Delta_{\text {Bun }_{G}}^{Z}$ is an isomorphism. 
3.2.3. We write

$$
\left(\Delta_{\mathrm{Bun}_{G}}\right)_{*}\left(\omega_{\mathrm{Bun}_{G}}\right) \simeq\left(\Delta_{\mathrm{Bun}_{G}}^{Z}\right)_{*} \circ\left(\operatorname{id}_{\mathrm{Bun}_{G}}^{Z}\right)_{*}\left(\omega_{\mathrm{Bun}_{G}}\right)
$$

In addition,

$\left(\Delta_{\mathrm{Bun}_{G}}\right) !\left(k_{\mathrm{Bun}_{G}}\right) \simeq\left(\Delta_{\mathrm{Bun}_{G}}^{Z}\right) ! \circ\left(\operatorname{id}_{\mathrm{Bun}_{G}}^{Z}\right)_{!}\left(k_{\mathrm{Bun}_{G}}\right) \simeq\left(\Delta_{\mathrm{Bun}_{G}}^{Z}\right) ! \circ\left(\operatorname{id}_{\mathrm{Bun}_{G}}^{Z}\right) !\left(\omega_{\mathrm{Bun}_{G}}\right)\left[-2 \operatorname{dim}\left(\operatorname{Bun}_{G}\right)\right]$, the latter isomorphism is due to the fact that $\operatorname{Bun}_{G}$ is smooth.

It is easy to see that

$$
\operatorname{triv!}(k) \simeq \operatorname{triv}_{*}(k)\left[-\operatorname{dim}\left(Z_{G}\right)\right]
$$

Hence,

$$
\left(\Delta_{\mathrm{Bun}_{G}}\right) !\left(k_{\mathrm{Bun}_{G}}\right) \simeq\left(\Delta_{\operatorname{Bun}_{G}}^{Z}\right) ! \circ\left(\operatorname{id}_{\mathrm{Bun}_{G}}^{Z}\right) *\left(\omega_{\mathrm{Bun}_{G}}\right)\left[-2 \operatorname{dim}\left(\operatorname{Bun}_{G}\right)-\operatorname{dim}\left(Z_{G}\right)\right] .
$$

Now, the morphism

$$
\Delta_{\mathrm{Bun}_{G}}^{Z}: \operatorname{Bun}_{G} \times B\left(Z_{G}\right) \rightarrow \operatorname{Bun}_{G} \times \operatorname{Bun}_{G}
$$

is schematic and separated. Hence, we obtain a natural transformation

$$
\left(\Delta_{\mathrm{Bun}_{G}}^{Z}\right) ! \rightarrow\left(\Delta_{\mathrm{Bun}_{G}}^{Z}\right)_{*} .
$$

Summarizing, we obtain a map

$$
\left(\Delta_{\mathrm{Bun}_{G}}\right) !\left(k_{\mathrm{Bun}_{G}}\right) \rightarrow\left(\Delta_{\mathrm{Bun}_{G}}\right)_{*}\left(\omega_{\mathrm{Bun}_{G}}\right)\left[-2 \operatorname{dim}\left(\operatorname{Bun}_{G}\right)-\operatorname{dim}\left(Z_{G}\right)\right] .
$$

3.2.4. From (3.3) and Sect. 2.1.3, we obtain a natural transformation:

$$
\text { Ps-Id } \operatorname{Bun}_{G}, ! \rightarrow \text { Ps- } \operatorname{Id}_{\mathrm{Bun}_{G}, \text { naive }}\left[-2 \operatorname{dim}\left(\operatorname{Bun}_{G}\right)-\operatorname{dim}\left(Z_{G}\right)\right]
$$

as functors $\mathrm{D}-\bmod \left(\operatorname{Bun}_{G}\right)_{\mathrm{co}} \rightarrow \mathrm{D}-\bmod \left(\operatorname{Bun}_{G}\right)$.

Let

$$
\mathrm{Ps}_{\mathrm{S}} \mathrm{Id}_{\mathrm{Bun}_{G}, \mathrm{diff}}: \mathrm{D}-\bmod \left(\mathrm{Bun}_{G}\right)_{\mathrm{co}} \rightarrow \mathrm{D}-\bmod \left(\operatorname{Bun}_{G}\right)
$$

denote the cone of the natural transformation (3.4).

3.2.5. We claim:

Proposition 3.2.6. The functor Ps-Id $\mathrm{Bun}_{G}$, diff admits a decreasing filtration, indexed by a poset, with subquotients being functors of the form

$$
\begin{aligned}
& \mathrm{D}-\bmod \left(\operatorname{Bun}_{G}\right)_{\mathrm{co}} \stackrel{\mathrm{CT}_{\mathrm{co} * *}^{\mu}}{\longrightarrow} \mathrm{D}-\bmod \left(\operatorname{Bun}_{M}^{\mu}\right)_{\mathrm{co}} \stackrel{\mathrm{Ps}^{-I_{1}} \stackrel{\mathrm{Bun}_{M}^{\mu}}{\longrightarrow}, \text { naive }}{\longrightarrow} \mathrm{D}-\bmod \left(\operatorname{Bun}_{M}^{\mu}\right) \stackrel{\mathrm{F}^{\mu, \mu^{\prime}}}{\longrightarrow} \\
& \rightarrow \mathrm{D}-\bmod \left(\operatorname{Bun}_{M}^{\mu^{\prime}}\right) \stackrel{\mathrm{Eis}_{*}^{\mu^{\prime},-}}{\longrightarrow} \mathrm{D}-\bmod \left(\operatorname{Bun}_{G}\right),
\end{aligned}
$$

for a proper parabolic $P$ with Levi quotient $M$, where $\mu, \mu^{\prime} \in \pi_{1}(M)$ and $\mathrm{F}^{\mu, \mu^{\prime}}$ is some functor $\mathrm{D}-\bmod \left(\operatorname{Bun}_{M}^{\mu}\right) \rightarrow \mathrm{D}-\bmod \left(\operatorname{Bun}_{M}^{\mu^{\prime}}\right)$. Furthermore, for a pair

$$
\left(U_{1} \stackrel{j_{1}}{\hookrightarrow} \operatorname{Bun}_{G}\right),\left(U_{2} \stackrel{j_{2}}{\hookrightarrow} \operatorname{Bun}_{G}\right) \in \mathrm{op}-\mathrm{qc}(G),
$$

the induced filtration on

is finite.

$$
j_{1}^{*} \circ \mathrm{Ps}-\operatorname{Id}_{\mathrm{Bun}_{G}, \operatorname{diff}} \circ\left(j_{2}\right)_{\mathrm{co}, *}
$$


The proof of Proposition 3.2.6 is analogous to that of Proposition 2.4.3 and will be given in [Sch].

As its geometric ingredient, instead of the stack $\widetilde{\operatorname{Bun}}_{P}$ appearing in the proof of Proposition 2.4.3, one uses a compactification of the morphism $\Delta_{\mathrm{Bun}_{G}}^{Z_{G}}$ which can be constructed using Vinberg's canonical semi-group of [Vi] attached to $G$.

\subsection{Pseudo-identity and cuspidality.}

3.3.1. As a consequence of Proposition 3.2.6, we obtain:

Corollary 3.3.2. The morphism (3.4) induces an isomorphism

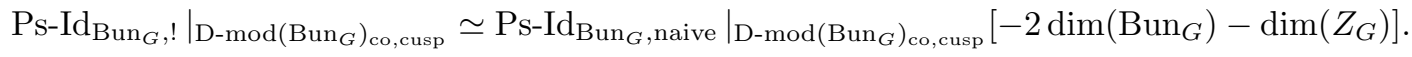

Proof. By the definition of D-mod $\left(\operatorname{Bun}_{G}\right)$, it is sufficient to show that for any $\left(U_{1} \stackrel{j_{1}}{\hookrightarrow} \operatorname{Bun}_{G}\right) \in$ op-qc $(G)$, the map (3.4) induces an isomorphism

$$
\begin{aligned}
j_{1}^{*} \circ \mathrm{Ps}-\left.\mathrm{Id}_{\mathrm{Bun}_{G}, !}\right|_{\mathrm{D}-\bmod \left(\operatorname{Bun}_{G}\right)_{\mathrm{co}, \text { cusp }}} \rightarrow & \\
& \rightarrow j_{1}^{*} \circ \mathrm{Ps}-\left.\mathrm{Id}_{\mathrm{Bun}_{G}, \text { naive }}\right|_{\mathrm{D}-\bmod \left(\operatorname{Bun}_{G}\right)_{\mathrm{co}, \text { cusp }}}\left[-2 \operatorname{dim}\left(\operatorname{Bun}_{G}\right)-\operatorname{dim}\left(Z_{G}\right)\right] .
\end{aligned}
$$

Let us take $U_{2}:=U_{G}$ as in Proposition 2.3.2. By Proposition 2.3.4, it suffices to show that for $\mathcal{F} \in \mathrm{D}-\bmod \left(\operatorname{Bun}_{G}\right)_{\text {co,cusp }}$

$$
j_{1}^{*} \circ \operatorname{Ps}_{-} \operatorname{Idd}_{\mathrm{Bun}_{G}, \operatorname{diff}} \circ\left(j_{2}\right)_{\mathrm{co}, *} \circ\left(j_{2}\right)_{\mathrm{co}}^{*}(\mathcal{F})=0 .
$$

However, this follows from Proposition 3.2.6:

Indeed, the object in question has a finite filtration, with subquotients isomorphic to

$$
j_{1}^{*} \circ \mathrm{Eis}_{*}^{\mu^{\prime},-} \circ \mathrm{F}^{\mu, \mu^{\prime}} \circ \mathrm{Ps}_{-} \mathrm{Id}_{\mathrm{Bun}_{M}^{\mu}, \text { naive }} \circ \mathrm{CT}_{\mathrm{co}, *}^{\mu} \circ\left(j_{2}\right)_{\mathrm{co}, *} \circ\left(j_{2}\right)_{\mathrm{co}}^{*}(\mathcal{F}),
$$

which, by Proposition 2.3.4, is isomorphic to

$$
j_{1}^{*} \circ \operatorname{Eis}_{*}^{\mu^{\prime},-} \circ \mathrm{F}^{\mu, \mu^{\prime}} \circ \operatorname{Ps}_{-} \operatorname{Id}_{\mathrm{Bun}_{M}^{\mu}, \text { naive }}\left(\mathrm{CT}_{\mathrm{co}, *}^{\mu}(\mathcal{F})\right),
$$

while $\mathrm{CT}_{\mathrm{co}, *}^{\mu}(\mathcal{F})=0$ by Corollary 2.4.7.

Corollary 3.3.3. The functor $\mathrm{Ps}_{-} \mathrm{Id}_{\mathrm{Bun}_{G}, !}$ induces an equivalence

$$
\mathrm{D}-\bmod \left(\operatorname{Bun}_{G}\right)_{\text {co,cusp }} \rightarrow \mathrm{D}-\bmod \left(\operatorname{Bun}_{G}\right)_{\text {cusp }} .
$$

Proof. Follows from Theorem 2.2.7 and Corollary 3.3.2.

3.3.4. The next assertion is a crucial step in the proof of Theorem 3.1.5:

Proposition 3.3.5. The functor $\mathrm{Ps}_{\mathrm{s}}-\mathrm{Id}_{\mathrm{Bun}_{G}, !}$ induces an isomorphism

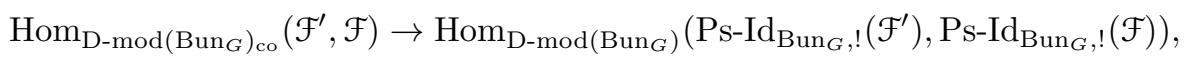

provided that $\mathcal{F}^{\prime} \in \mathrm{D}-\bmod \left(\mathrm{Bun}_{G}\right)_{\mathrm{co}, \mathrm{cusp}}$.

\subsection{Proof of Proposition 3.3.5.}


3.4.1. Let us first assume that $\mathcal{F}$ has the form $j_{\mathrm{co}, *}\left(\mathcal{F}_{U}\right)$ for some $\left(U \stackrel{j}{\hookrightarrow} \operatorname{Bun}_{G}\right) \in$ op-qc $(G)$.

Consider the commutative diagram

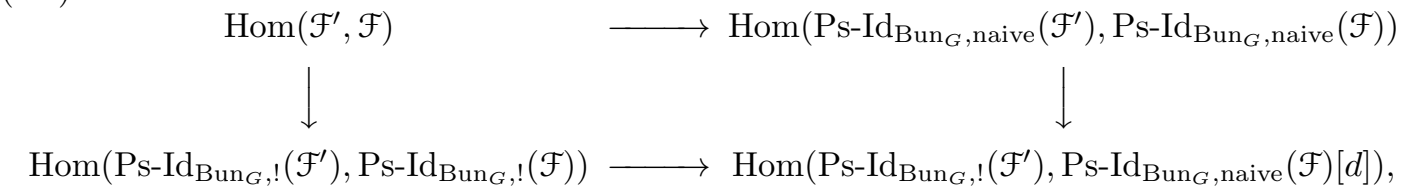

where $d=-2 \operatorname{dim}\left(\operatorname{Bun}_{G}\right)-\operatorname{dim}\left(Z_{G}\right)$.

We need to show that the left vertical arrow is an isomorphism. We will do so by showing that all the other arrows are isomorphisms.

3.4.2. First, we claim that upper horizontal arrow in (3.5) is an isomorphism for any $\mathcal{F}^{\prime} \in$ $\mathrm{D}-\bmod \left(\operatorname{Bun}_{G}\right)_{\text {co }}$ and $\mathcal{F}=j_{*}\left(\mathcal{F}_{U}\right)$. Indeed, the map in question fits into a commutative diagram

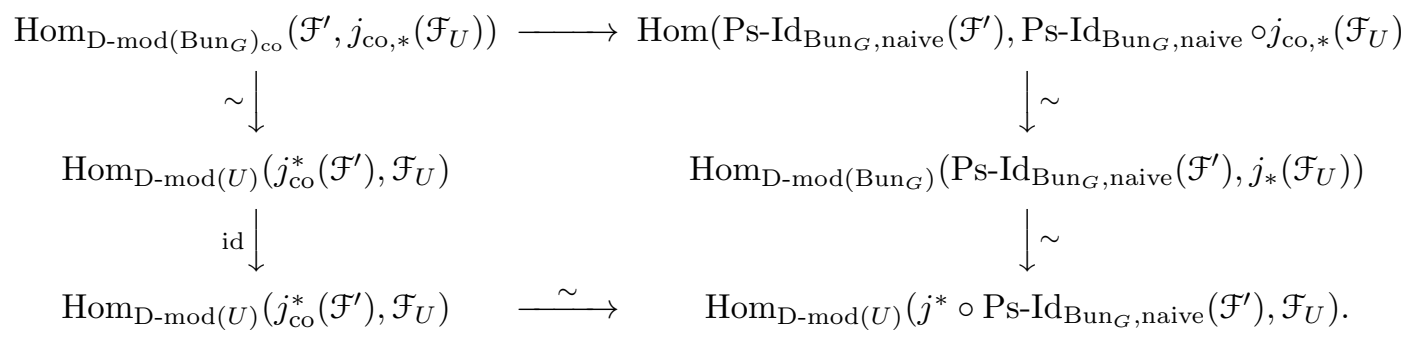

3.4.3. The right vertical arrow in (3.5) is an isomorphism by Corollary 3.3.2.

To show that the lower horizontal arrow is an isomorphism, using Corollary 3.3.3, it suffices to show that for any $\mathcal{F}^{\prime \prime} \in \mathrm{D}-\bmod \left(\operatorname{Bun}_{G}\right)_{\text {cusp }}$, we have

$$
\operatorname{Hom}_{\mathrm{D}-\bmod \left(\operatorname{Bun}_{G}\right)}\left(\mathcal{F}^{\prime \prime}, \operatorname{Ps}_{\mathrm{S}} \operatorname{Id}_{\mathrm{Bun}_{G}, \operatorname{diff}} \circ j_{\mathrm{co}, *}\left(\mathcal{F}_{U}\right)\right)=0 .
$$

By Proposition 2.3.2,

$$
\begin{aligned}
& \operatorname{Hom}_{\mathrm{D}-\bmod \left(\operatorname{Bun}_{G}\right)}\left(\mathcal{F}^{\prime \prime}, \operatorname{Ps}-\operatorname{Id}_{\mathrm{Bun}_{G}, \operatorname{diff}} \circ j_{\mathrm{co}, *}\left(\mathcal{F}_{U}\right)\right) \simeq \\
& \simeq \operatorname{Hom}_{\mathrm{D}-\bmod \left(\mathcal{U}_{G}\right)}\left(j_{G}^{*}\left(\mathcal{F}^{\prime \prime}\right), \jmath_{G}^{*} \circ \mathrm{Ps}_{\mathrm{s}} \operatorname{Id}_{\mathrm{Bun}}, \operatorname{diff} \circ j_{\mathrm{co}, *}\left(\mathcal{F}_{U}\right)\right) \text {. }
\end{aligned}
$$

Applying Proposition 3.2.6, we obtain that it suffices to show that for $\mathcal{F}^{\prime \prime} \in \mathrm{D}$-mod$\left(\operatorname{Bun}_{G}\right)_{\text {cusp }}$

$$
\operatorname{Hom}_{\mathrm{D}-\bmod \left(\mathcal{U}_{G}\right)}\left(\jmath_{G}^{*}\left(\mathcal{F}^{\prime \prime}\right), \jmath_{G}^{*} \circ \operatorname{Eis}_{*}^{\mu^{\prime},-} \circ \mathrm{F}^{\mu, \mu^{\prime}} \circ \operatorname{Ps}_{-} \operatorname{Id}_{\mathrm{Bun}_{M}^{\mu}, \text { naive }} \circ \mathrm{CT}_{\mathrm{co}, *}^{\mu} \circ j_{\mathrm{co}, *}\left(\mathcal{F}_{U}\right)\right)=0
$$

which by Proposition 2.3.2 is equivalent to

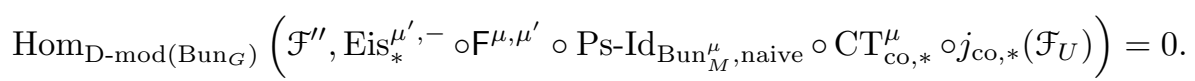

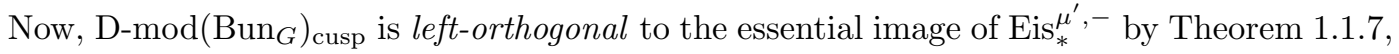
implying the desired vanishing. 
3.4.4. We will now reduce the assertion of Proposition 3.3.5 to the situation of Sect. 3.4.1.

Let us recall that according to [DrGa2, Theorem 4.1.8], any element $\left(U \stackrel{j}{\hookrightarrow} \operatorname{Bun}_{G}\right) \in$ op-qc $(G)$ is contained in one which is co-truncative. See [DrGa2, Sect. 3.8] for what it means for an open substack to be co-truncative. In particular, the open substack $\mathcal{U}_{G}$ of Proposition 2.3.2 can be enlarged so that it is co-trunactive.

Recall also that for a co-truncative open substack $U \stackrel{j}{\hookrightarrow} \operatorname{Bun}_{G}$, the functor $j_{\mathrm{co}, *}$ has a (continuous) right adjoint, denoted $j$ ?, see [DrGa2, Sect. 4.3].

Any $\mathcal{F} \in \mathrm{D}-\bmod \left(\operatorname{Bun}_{G}\right)_{\text {co }}$ fits into an exact triangle

$$
\mathcal{F}_{1} \rightarrow \mathcal{F} \rightarrow j_{\mathrm{co}, *} \circ j^{?}(\mathcal{F}),
$$

where $j^{?}\left(\mathcal{F}_{1}\right)=0$ by Lemma 1.5.2.

We take $U$ to contain the substack $\mathcal{U}_{G}$ as in Proposition 2.3.2, and assume that it is cotruncative. In view of Proposition 2.3.4 and Corollary 3.3.3, it remains to show that if $j^{?}(\mathcal{F})=0$, then

$$
\operatorname{Hom}_{\mathrm{D}-\bmod \left(\operatorname{Bun}_{G}\right)}\left(\mathcal{F}^{\prime \prime}, \operatorname{Ps}_{-I_{d}} \operatorname{Bun}_{G}, !(\mathcal{F})\right)=0, \quad \mathcal{F}^{\prime \prime} \in \mathrm{D}-\bmod \left(\operatorname{Bun}_{G}\right)_{\text {cusp }} .
$$

By Proposition 2.3.2, it suffices to show that

$$
j^{?}(\mathcal{F})=0 \Rightarrow j^{*} \circ \operatorname{Ps}-\operatorname{Id}_{\mathrm{Bun}_{G}, !}(\mathcal{F})=0 .
$$

However, this follows from (the nearly tautological) [Ga2, Corollary 6.6.3].

\section{The strange functional equation and proof of the Equivalence}

In this section we will carry out the two main tasks of this paper: we will prove the strange functional equation (Theorem 4.1.2 below) and finish the proof of Theorem 3.1.5 (that says that the functor Ps- $\operatorname{Id}_{\mathrm{Bun}_{G}, !}$ is an equivalence).

4.1. The strange functional equation. In this subsection we will study the behavior of the functor Ps- $\mathrm{Id}_{\mathrm{Bun}_{G}, !}$ on the subcategory

$$
\mathrm{D}-\bmod \left(\operatorname{Bun}_{G}\right)_{\mathrm{co}, \text { Eis }} \subset \mathrm{D}-\bmod \left(\operatorname{Bun}_{G}\right)_{\mathrm{co}} \text {. }
$$

4.1.1. First, we have the following "strange" result:

Theorem 4.1.2. For a parabolic $P$ and its opposite $P^{-}$we have a canonical isomorphism of functors

$$
\text { Eis } !_{!} \circ \operatorname{Ps}_{-}-I_{\mathrm{Bun}_{M}, !} \simeq \operatorname{Ps}_{-I_{\mathrm{Bun}_{G}, !} \circ \text { Eis }_{\mathrm{co}, *}^{-}} \text {. }
$$

Proof. Both sides are continuous functors

$$
\mathrm{D}-\bmod \left(\operatorname{Bun}_{M}\right)_{\mathrm{co}, *} \rightarrow \mathrm{D}-\bmod \left(\operatorname{Bun}_{G}\right),
$$

that correspond to objects of

$$
\mathrm{D}-\bmod \left(\operatorname{Bun}_{M} \times \operatorname{Bun}_{G}\right)
$$

under the identification

$$
\begin{aligned}
\text { Funct }_{\text {cont }}\left(\mathrm{D}-\bmod \left(\operatorname{Bun}_{M}\right)_{\mathrm{co}},\right. & \left.\mathrm{D}-\bmod \left(\operatorname{Bun}_{G}\right)\right) \simeq\left(\mathrm{D}-\bmod \left(\operatorname{Bun}_{M}\right)_{\mathrm{co}}\right)^{\vee} \otimes \mathrm{D}-\bmod \left(\operatorname{Bun}_{G}\right) \simeq \\
& \simeq \mathrm{D}-\bmod \left(\operatorname{Bun}_{M}\right) \otimes \mathrm{D}-\bmod \left(\operatorname{Bun}_{G}\right) \simeq \mathrm{D}-\bmod \left(\operatorname{Bun}_{M} \times \operatorname{Bun}_{G}\right),
\end{aligned}
$$

We claim that both objects identify canonically with

$$
\left((\mathrm{q} \times \mathrm{p}) \circ \Delta_{\mathrm{Bun}_{P}}\right) !\left(k_{\mathrm{Bun}_{P}}\right),
$$


where the map in the formula is the same as

$$
\operatorname{Bun}_{P} \stackrel{\mathrm{q} \times \mathrm{p}}{\longrightarrow} \operatorname{Bun}_{M} \times \operatorname{Bun}_{G} .
$$

The functor Eis! $\circ$ Ps-Id $\mathrm{Bun}_{M}, !$ corresponds to the object, obtained by applying the functor $\left(\mathrm{Id}_{\mathrm{D}-\bmod \left(\operatorname{Bun}_{M}\right)} \otimes \operatorname{Eis!}\right): \mathrm{D}-\bmod \left(\operatorname{Bun}_{M}\right) \otimes \mathrm{D}-\bmod \left(\operatorname{Bun}_{M}\right) \rightarrow \mathrm{D}-\bmod \left(\operatorname{Bun}_{M}\right) \otimes \mathrm{D}-\bmod \left(\operatorname{Bun}_{G}\right)$ to

$$
\left(\Delta_{\operatorname{Bun}_{M}}\right) !\left(k_{\operatorname{Bun}_{M}}\right) \in \mathrm{D}-\bmod \left(\operatorname{Bun}_{M} \times \operatorname{Bun}_{M}\right) \simeq \mathrm{D}-\bmod \left(\operatorname{Bun}_{M}\right) \otimes \mathrm{D}-\bmod \left(\operatorname{Bun}_{M}\right) .
$$

The functor $\operatorname{Id}_{\mathrm{D}-\bmod \left(\operatorname{Bun}_{M}\right)} \otimes$ Eis! is left adjoint to the functor

$$
\operatorname{Id}_{\mathrm{D}-\bmod \left(\operatorname{Bun}_{M}\right)} \otimes \mathrm{CT}_{*} \simeq\left(\mathrm{id}_{\mathrm{Bun}_{M}} \times \mathrm{q}\right)_{*} \circ\left(\operatorname{id}_{\mathrm{Bun}_{M}} \times \mathrm{p}\right) !,
$$

and hence is the !-Eisenstein series functor for the group $M \times G$ with respect to the parabolic $M \times P$. I.e., it is given by

$$
\left(\operatorname{id}_{\operatorname{Bun}_{M}} \times \mathrm{p}\right) ! \times\left(\operatorname{id}_{\mathrm{Bun}_{M}} \times \mathbf{q}\right)^{*},
$$

when applied to holonomic objects.

Base change along the diagram

shows that

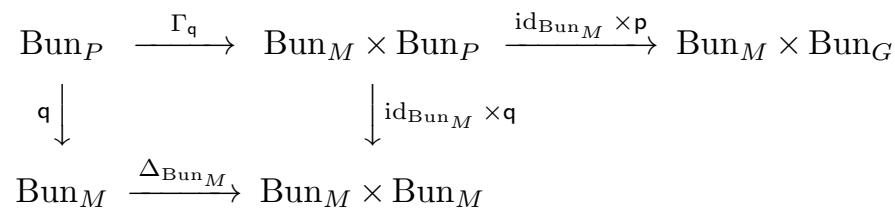

$$
\left(\operatorname{id}_{\operatorname{Bun}_{M}} \times \mathrm{p}\right) ! \times\left(\operatorname{id}_{\mathrm{Bun}_{M}} \times \mathrm{q}\right)^{*} \circ\left(\Delta_{\mathrm{Bun}_{M}}\right)_{!}\left(k_{\mathrm{Bun}_{M}}\right) \simeq\left((\mathrm{q} \times \mathrm{p}) \circ \Delta_{\mathrm{Bun}_{P}}\right) !\left(k_{\mathrm{Bun}_{P}}\right),
$$

as required.

The functor Ps-Id $\mathrm{Bun}_{G}, ! \circ \mathrm{Eis}_{\mathrm{co}, *}^{-}$corresponds to the object, obtained by applying the functor $\left(\left(\operatorname{Eis}_{\mathrm{co}, *}^{-}\right)^{\vee} \otimes \operatorname{Id}_{\mathrm{D}-\bmod \left(\operatorname{Bun}_{G}\right)}\right):$

$$
\mathrm{D}-\bmod \left(\operatorname{Bun}_{G}\right) \otimes \mathrm{D}-\bmod \left(\mathrm{Bun}_{G}\right) \rightarrow \mathrm{D}-\bmod \left(\operatorname{Bun}_{M}\right) \otimes \mathrm{D}-\bmod \left(\operatorname{Bun}_{G}\right)
$$

to the object

$$
\left(\Delta_{\mathrm{Bun}_{G}}\right) !\left(k_{\mathrm{Bun}_{G}}\right) \in \mathrm{D}-\bmod \left(\operatorname{Bun}_{G} \times \mathrm{Bun}_{G}\right) \simeq \mathrm{D}-\bmod \left(\operatorname{Bun}_{G}\right) \otimes \mathrm{D}-\bmod \left(\operatorname{Bun}_{G}\right) .
$$

We have:

$$
\left(\operatorname{Eis}_{\mathrm{co}, *}^{-}\right)^{\vee} \simeq \mathrm{CT}_{*}^{-},
$$

and we recall that by Theorem 1.1.7

$$
\mathrm{CT}_{*}^{-} \simeq \mathrm{CT}_{!}:=\bigoplus_{\mu} \mathrm{CT}_{!}^{\mu},
$$

where $\mathrm{CT}_{!}^{\mu}$ is the left adjoint of $\mathrm{Eis}_{*}^{\mu}$.

Since $\mathrm{CT}_{!}^{\mu}$ is the left adjoint of $\operatorname{Eis}_{*}^{\mu}$, we obtain that $\mathrm{CT}_{!}^{\mu} \otimes \mathrm{Id}_{\mathrm{D}-\bmod \left(\mathrm{Bun}_{G}\right)}$ is the left adjoint of $\operatorname{Eis}_{*}^{\mu} \otimes \operatorname{Id}_{\mathrm{D}-\bmod \left(\operatorname{Bun}_{G}\right)}$, i.e., is the !-constant term functor for the group $G \times G$ with respect to the parabolic $P \times G$. Hence,

$$
\mathrm{CT}_{!}^{\mu} \otimes \operatorname{Id}_{\mathrm{D}-\bmod \left(\operatorname{Bun}_{G}\right)} \simeq\left(\mathrm{q}^{\mu} \times \operatorname{id}_{\mathrm{Bun}_{G}}\right) ! \circ\left(\mathrm{p}^{\mu} \times \mathrm{id}_{\mathrm{Bun}_{G}}\right)^{*},
$$

when appied to holonomic objects (the superscipt $\mu$ indicates that we are taking only the $\mu$-connected component of $\left.\operatorname{Bun}_{P}\right)$. 
Taking the direct sum over $\mu$, we thus obtain

$$
\left(\operatorname{Eis}_{\mathrm{co}, *}^{-}\right)^{\vee} \otimes \operatorname{Id}_{\mathrm{D}-\bmod \left(\operatorname{Bun}_{G}\right)} \simeq\left(\mathrm{q} \times \operatorname{id}_{\mathrm{Bun}_{G}}\right) ! \circ\left(\mathrm{p} \times \operatorname{id}_{\mathrm{Bun}_{G}}\right)^{*},
$$

when applied to holonomic objects.

Now, base change along the diagram

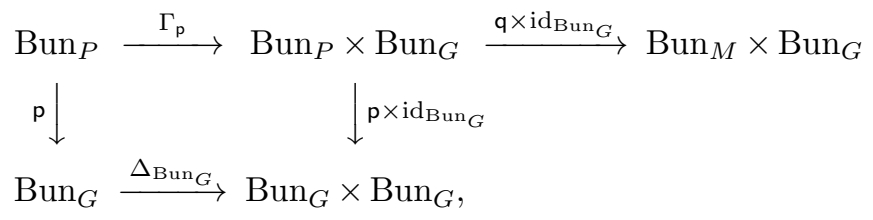

shows that

$$
\left(\mathrm{q} \times \operatorname{id}_{\mathrm{Bun}_{G}}\right) ! \circ\left(\mathrm{p} \times \operatorname{id}_{\mathrm{Bun}_{G}}\right)^{*} \circ\left(\Delta_{\mathrm{Bun}_{G}}\right) !\left(k_{\mathrm{Bun}_{G}}\right) \simeq\left((\mathrm{q} \times \mathrm{p}) \circ \Delta_{\mathrm{Bun}_{P}}\right) !\left(k_{\mathrm{Bun}_{P}}\right),
$$

as required.

4.1.3. By passing to dual functors in the isomorphism

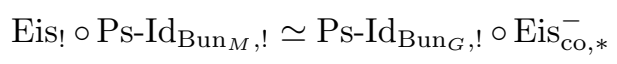

of Theorem 4.1.2, we obtain:

Corollary 4.1.4. There is a canonical isomorphism

$$
\mathrm{Ps}-\mathrm{Id}_{\mathrm{Bun}_{M}, !} \circ \mathrm{CT}_{\mathrm{co}, ?} \simeq \mathrm{CT}_{*}^{-} \circ \mathrm{Ps}_{-}-\mathrm{Id}_{\mathrm{Bun}_{G}, !} \cdot
$$

4.1.5. Consider now the commutative diagram:

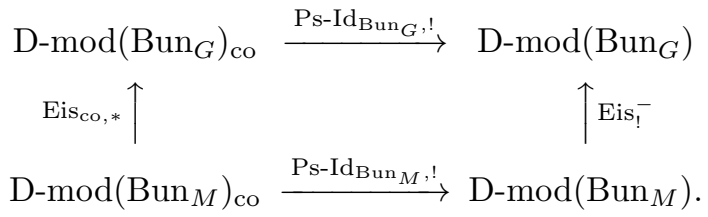

By passing to the right adjoint functors along the vertical arrows, we obtain a natural transformation

$$
\mathrm{Ps}_{\mathrm{S}}-\mathrm{Id}_{\mathrm{Bun}_{M}, !} \circ \mathrm{CT}_{\mathrm{co}, ?} \rightarrow \mathrm{CT}_{*}^{-} \circ \mathrm{Ps}_{\mathrm{s}}-\mathrm{Id}_{\mathrm{Bun}_{G}, !} \cdot
$$

We now claim:

Proposition 4.1.6. The map (4.4) equals the map (4.2), and, in particular, is an isomorphism.

4.2. Proof of Proposition 4.1.6. The proof of the proposition is not a formal manipulation, as its statement involves the isomorphism of Theorem 4.1.2 for the two different parabolics, namely, $P$ and $P^{-}$. The corresponding geometric input is provided by Lemma 4.2 .3 below.

4.2.1. Let us identify

$$
\mathrm{CT}_{*}^{-} \simeq \mathrm{CT}_{!} \text {and } \mathrm{Eis}_{\mathrm{co}, *} \simeq\left(\mathrm{CT}_{!}^{-}\right)^{\vee}
$$

via Theorem 1.1.7.

Then the map

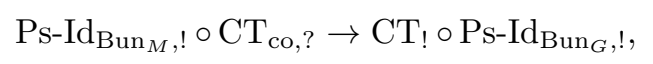


corresponding to (4.4), equals by definition the composition

$$
\begin{aligned}
& \mathrm{Ps}_{-} \mathrm{Id}_{\mathrm{Bun}_{M}, !} \circ \mathrm{CT}_{\mathrm{co}, ?} \rightarrow \mathrm{CT}_{!} \circ \mathrm{Eis}_{!}^{-} \circ \mathrm{Ps}_{-}-\operatorname{Id}_{\mathrm{Bun}_{M}, !} \circ \mathrm{CT}_{\mathrm{co}, ?} \stackrel{(4.3)}{\simeq} \\
& \simeq \mathrm{CT}_{!} \circ \mathrm{Ps}_{-} \mathrm{Id}_{\mathrm{Bun}_{G}, !} \circ \mathrm{Eis}_{\mathrm{co}, *} \circ \mathrm{CT}_{\mathrm{co}, ?} \simeq \mathrm{CT}_{!} \circ \mathrm{Ps}_{\mathrm{s}}-\mathrm{Id}_{\mathrm{Bun}_{G}, !} \circ\left(\mathrm{CT}_{!}^{-}\right)^{\vee} \circ\left(\mathrm{Eis}_{!}\right)^{\vee}= \\
& =\mathrm{CT}_{!} \circ \mathrm{Ps}_{\mathrm{s}}-\mathrm{Id}_{\mathrm{Bun}_{G}, !} \circ\left(\text { Eis }_{!} \circ \mathrm{CT}_{!}^{-}\right)^{\vee} \rightarrow \mathrm{CT}_{!} \circ \mathrm{Ps}_{\mathrm{S}}-\mathrm{Id}_{\mathrm{Bun}_{G}, !},
\end{aligned}
$$

where the first arrows comes from the unit of the (Eis! $\left.{ }^{-}, \mathrm{CT}_{!}\right)$-adjunction, and the last arrow comes from the co-unit of the (Eis!, $\mathrm{CT}_{!}^{-}$)-adjunction.

This corresponds to the following map of objects in D-mod( $\left(\operatorname{Bun}_{G} \times \operatorname{Bun}_{M}\right)$ :

$$
\begin{aligned}
& \left(\operatorname{Eis!} \otimes \operatorname{Id}_{\mathrm{D}-\bmod \left(\operatorname{Bun}_{M}\right)}\right) \circ\left(\Delta_{\mathrm{D}-\bmod \left(\operatorname{Bun}_{M}\right)}\right) !\left(k_{\mathrm{Bun}_{M}}\right) \rightarrow \\
& \rightarrow\left(\operatorname{Eis!} \otimes\left(\mathrm{CT}_{!} \circ \operatorname{Eis}_{!}^{-}\right)\right) \circ\left(\Delta_{\mathrm{D}-\bmod \left(\operatorname{Bun}_{M}\right)}\right) !\left(k_{\mathrm{Bun}_{M}}\right)= \\
& =\left(\operatorname{Eis}_{!} \otimes \mathrm{CT}_{!}\right) \circ\left(\operatorname{Id}_{\mathrm{D}-\bmod \left(\operatorname{Bun}_{M}\right)} \otimes \operatorname{Eis}_{!}^{-}\right) \circ\left(\Delta_{\mathrm{D}-\bmod \left(\operatorname{Bun}_{M}\right)}\right) !\left(k_{\mathrm{Bun}_{M}}\right) \simeq \\
& \simeq\left(\operatorname{Eis!} \otimes \mathrm{CT}_{!}\right) \circ\left(\mathrm{CT}_{!}^{-} \circ \mathrm{Id}_{\mathrm{D}-\bmod \left(\operatorname{Bun}_{G}\right)}\right) \circ\left(\Delta_{\mathrm{D}-\bmod \left(\operatorname{Bun}_{G}\right)}\right) !\left(k_{\mathrm{Bun}_{G}}\right)= \\
& =\left(\left(\text { Eis! } !_{1} \mathrm{CT}_{!}^{-}\right) \otimes \mathrm{CT} !\right) \circ\left(\Delta_{\mathrm{D}-\bmod \left(\mathrm{Bun}_{G}\right)}\right) !\left(k_{\mathrm{Bun}_{G}}\right) \rightarrow \\
& \rightarrow\left(\mathrm{Id}_{\mathrm{D}-\bmod \left(\mathrm{Bun}_{G}\right)} \otimes \mathrm{CT} !\right) \circ\left(\Delta_{\mathrm{D}-\bmod \left(\operatorname{Bun}_{G}\right)}\right) !\left(k_{\mathrm{Bun}_{G}}\right),
\end{aligned}
$$

where the isomorphism between the $3 \mathrm{rd}$ and the 4 th lines is

$$
\begin{aligned}
\left(\operatorname{Id}_{\mathrm{D}-\bmod \left(\operatorname{Bun}_{M}\right)} \otimes \operatorname{Eis}_{!}^{-}\right) \circ( & \left.\Delta_{\mathrm{D}-\bmod \left(\operatorname{Bun}_{M}\right)}\right) !\left(k_{\mathrm{Bun}_{M}}\right) \simeq \\
& \simeq\left(\left(\mathrm{q}^{-} \times \mathrm{p}^{-}\right) \circ \Delta_{\mathrm{Bun}_{P^{-}}}\right) !\left(k_{\mathrm{Bun}_{P^{-}}}\right) \simeq \\
& \simeq\left(\mathrm{CT}_{!}^{-} \circ \operatorname{Id}_{\mathrm{D}-\bmod \left(\operatorname{Bun}_{G}\right)}\right) \circ\left(\Delta_{\mathrm{D}-\bmod \left(\operatorname{Bun}_{G}\right)}\right) !\left(k_{\mathrm{Bun}_{G}}\right),
\end{aligned}
$$

used in the proof of Theorem 4.1.2.

The assertion of the proposition amounts to showing that the composed map in (4.5) equals

$\left(\operatorname{Eis!} \otimes \operatorname{Id}_{\mathrm{D}-\bmod \left(\operatorname{Bun}_{M}\right)}\right) \circ\left(\Delta_{\mathrm{D}-\bmod \left(\operatorname{Bun}_{M}\right)}\right) !\left(k_{\mathrm{Bun}_{M}}\right) \simeq$

$$
\begin{aligned}
\simeq\left((\mathrm{p} \times \mathrm{q}) \circ \Delta_{\mathrm{Bun}_{P}}\right) !\left(k_{\mathrm{Bun}_{P}}\right) & \simeq \\
& \simeq\left(\operatorname{Id}_{\mathrm{D}-\bmod \left(\operatorname{Bun}_{G}\right)} \otimes \mathrm{CT} !\right) \circ\left(\Delta_{\mathrm{D}-\bmod \left(\operatorname{Bun}_{G}\right)}\right) !\left(k_{\mathrm{Bun}_{G}}\right) .
\end{aligned}
$$

4.2.2. The geometric input is provided by the following assertion, proved at the end of this subsection:

Lemma 4.2.3. The following diagram commutes:

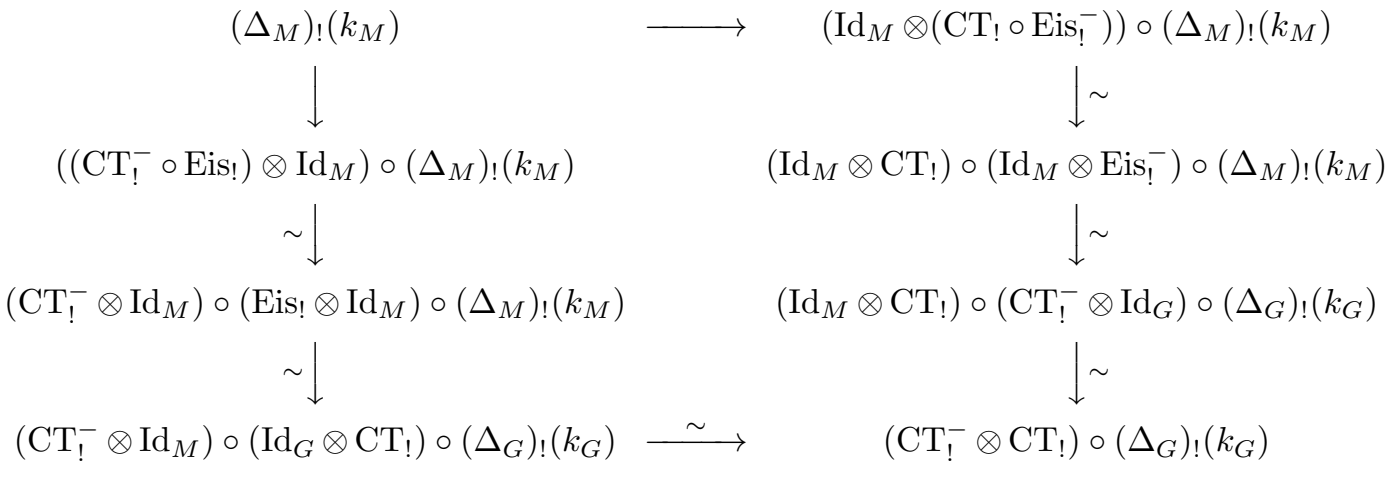

where we use short-hand $\operatorname{Id}_{M}, \Delta_{M}, k_{M}$ for $\operatorname{Id}_{\mathrm{D}-\bmod \left(\operatorname{Bun}_{M}\right)}, \Delta_{\mathrm{Bun}_{M}}$ and $k_{\mathrm{Bun}_{M}}$, respectively, and similarly for $G$. 
Using the lemma, we rewrite the map in (4.5) as follows:

$$
\begin{aligned}
& \left(\operatorname{Eis!} \otimes \operatorname{Id}_{\mathrm{D}-\bmod \left(\operatorname{Bun}_{M}\right)}\right) \circ\left(\Delta_{\mathrm{D}-\bmod \left(\operatorname{Bun}_{M}\right)}\right) !\left(k_{\mathrm{Bun}_{M}}\right) \rightarrow \\
& \rightarrow\left(\left(\text { Eis! } \circ \mathrm{CT}_{!}^{-} \circ \operatorname{Eis}_{!}\right) \otimes \mathrm{Id}_{\mathrm{D}-\bmod \left(\operatorname{Bun}_{M}\right)}\right) \circ\left(\Delta_{\mathrm{D}-\bmod \left(\operatorname{Bun}_{M}\right)}\right) !\left(k_{\mathrm{Bun}_{M}}\right)= \\
& =\left(\left(\operatorname{Eis}_{!} \circ \mathrm{CT}_{!}^{-}\right) \otimes \mathrm{Id}_{\mathrm{D}-\bmod \left(\operatorname{Bun}_{M}\right)}\right) \circ\left(\operatorname{Eis}_{!} \otimes \operatorname{Id}_{\mathrm{D}-\bmod \left(\operatorname{Bun}_{M}\right)}\right) \circ\left(\Delta_{\mathrm{D}-\bmod \left(\operatorname{Bun}_{M}\right)}\right) !\left(k_{\mathrm{Bun}_{M}}\right) \simeq \\
& \simeq\left(\left(\operatorname{Eis}_{!} \circ \mathrm{CT}_{!}^{-}\right) \otimes \mathrm{Id}_{\mathrm{D}-\bmod \left(\operatorname{Bun}_{M}\right)}\right) \circ\left(\operatorname{Id}_{\mathrm{D}-\bmod \left(\operatorname{Bun}_{G}\right)} \otimes \mathrm{CT} !\right) \circ\left(\Delta_{\mathrm{D}-\bmod \left(\operatorname{Bun}_{G}\right)}\right) !\left(k_{\mathrm{Bun}_{G}}\right) \rightarrow \\
& \rightarrow\left(\mathrm{Id}_{\mathrm{D}-\bmod \left(\operatorname{Bun}_{G}\right)} \otimes \mathrm{CT} !\right) \circ\left(\Delta_{\mathrm{D}-\bmod \left(\operatorname{Bun}_{G}\right)}\right) !\left(k_{\mathrm{Bun}_{G}}\right),
\end{aligned}
$$

and further as

$$
\begin{aligned}
& \left(\operatorname{Eis} ! \otimes \operatorname{Id}_{\mathrm{D}-\bmod \left(\operatorname{Bun}_{M}\right)}\right) \circ\left(\Delta_{\mathrm{D}-\bmod \left(\operatorname{Bun}_{M}\right)}\right) !\left(k_{\mathrm{Bun}_{M}}\right) \rightarrow \\
& \rightarrow\left(\left(\text { Eis! } \circ \mathrm{CT}_{!}^{-} \circ \operatorname{Eis}_{!}\right) \otimes \operatorname{Id}_{\mathrm{D}-\bmod \left(\operatorname{Bun}_{M}\right)}\right) \circ\left(\Delta_{\mathrm{D}-\bmod \left(\operatorname{Bun}_{M}\right)}\right) !\left(k_{\mathrm{Bun}_{M}}\right)= \\
& =\left(\left(\operatorname{Eis} !_{\mathrm{S}} \circ \mathrm{CT}_{!}^{-}\right) \otimes \operatorname{Id}_{\mathrm{D}-\bmod \left(\operatorname{Bun}_{M}\right)}\right) \circ\left(\operatorname{Eis!} \otimes \operatorname{Id}_{\mathrm{D}-\bmod \left(\operatorname{Bun}_{M}\right)}\right) \circ\left(\Delta_{\mathrm{D}-\bmod \left(\operatorname{Bun}_{M}\right)}\right) !\left(k_{\mathrm{Bun}_{M}}\right) \rightarrow \\
& \rightarrow\left(\operatorname{Eis!} \otimes \operatorname{Id}_{\mathrm{D}-\bmod \left(\operatorname{Bun}_{M}\right)}\right) \circ\left(\Delta_{\mathrm{D}-\bmod \left(\operatorname{Bun}_{M}\right)}\right) !\left(k_{\mathrm{Bun}_{M}}\right) \simeq \\
& \simeq\left(\mathrm{Id}_{\mathrm{D}-\bmod \left(\operatorname{Bun}_{G}\right)} \otimes \mathrm{CT} !\right) \circ\left(\Delta_{\mathrm{D}-\bmod \left(\operatorname{Bun}_{G}\right)}\right) !\left(k_{\mathrm{Bun}_{G}}\right) \text {. }
\end{aligned}
$$

However, the composition

$$
\begin{aligned}
& \left(\operatorname{Eis!} \otimes \operatorname{Id}_{\mathrm{D}-\bmod \left(\operatorname{Bun}_{M}\right)}\right) \circ\left(\Delta_{\mathrm{D}-\bmod \left(\operatorname{Bun}_{M}\right)}\right) !\left(k_{\mathrm{Bun}_{M}}\right) \rightarrow \\
& \rightarrow\left(\left(\text { Eis! }_{0} \circ \mathrm{CT}_{!}^{-} \circ \operatorname{Eis!}\right) \otimes \operatorname{Id}_{\mathrm{D}-\bmod \left(\operatorname{Bun}_{M}\right)}\right) \circ\left(\Delta_{\mathrm{D}-\bmod \left(\operatorname{Bun}_{M}\right)}\right) !\left(k_{\mathrm{Bun}_{M}}\right)= \\
& =\left(\left(\operatorname{Eis!} \circ \mathrm{CT}_{!}^{-}\right) \otimes \operatorname{Id}_{\mathrm{D}-\bmod \left(\operatorname{Bun}_{M}\right)}\right) \circ\left(\operatorname{Eis!} \otimes \operatorname{Id}_{\mathrm{D}-\bmod \left(\operatorname{Bun}_{M}\right)}\right) \circ\left(\Delta_{\mathrm{D}-\bmod \left(\operatorname{Bun}_{M}\right)}\right) !\left(k_{\mathrm{Bun}_{M}}\right) \rightarrow \\
& \rightarrow\left(\operatorname{Eis!} \otimes \operatorname{Id}_{\mathrm{D}-\bmod \left(\operatorname{Bun}_{M}\right)}\right) \circ\left(\Delta_{\mathrm{D}-\bmod \left(\operatorname{Bun}_{M}\right)}\right) !\left(k_{\mathrm{Bun}_{M}}\right)
\end{aligned}
$$

is the identity map, as it is induced by the map

$$
\text { Eis! } \rightarrow \text { Eis! } \circ \mathrm{CT}_{!}^{-} \circ \text { Eis! } \rightarrow \text { Eis! }
$$

comprised by the unit and co-unit of the (Eis! $\mathrm{CT}_{!}^{-}$)-adjunction, and the assertion follows.

4.2.4. Proof of Lemma 4.2.3. Let us recall from [DrGa3, Sect. 1.3.2] that the unit for the $\left(\right.$ Eis!, $\left.\mathrm{CT}_{!}^{-}\right)$can be described as follows. The functor

$$
\mathrm{CT}_{!} \circ \mathrm{Eis}_{!}^{-}: \mathrm{D}-\bmod \left(\mathrm{Bun}_{M}\right) \rightarrow \mathrm{D}-\bmod \left(\mathrm{Bun}_{M}\right)
$$

is given by

$$
(\mathrm{q}) ! \circ(\mathrm{p})^{*} \circ\left(\mathrm{p}^{-}\right) ! \circ\left(\mathrm{q}^{-}\right)^{*},
$$


which by base change along the diagram

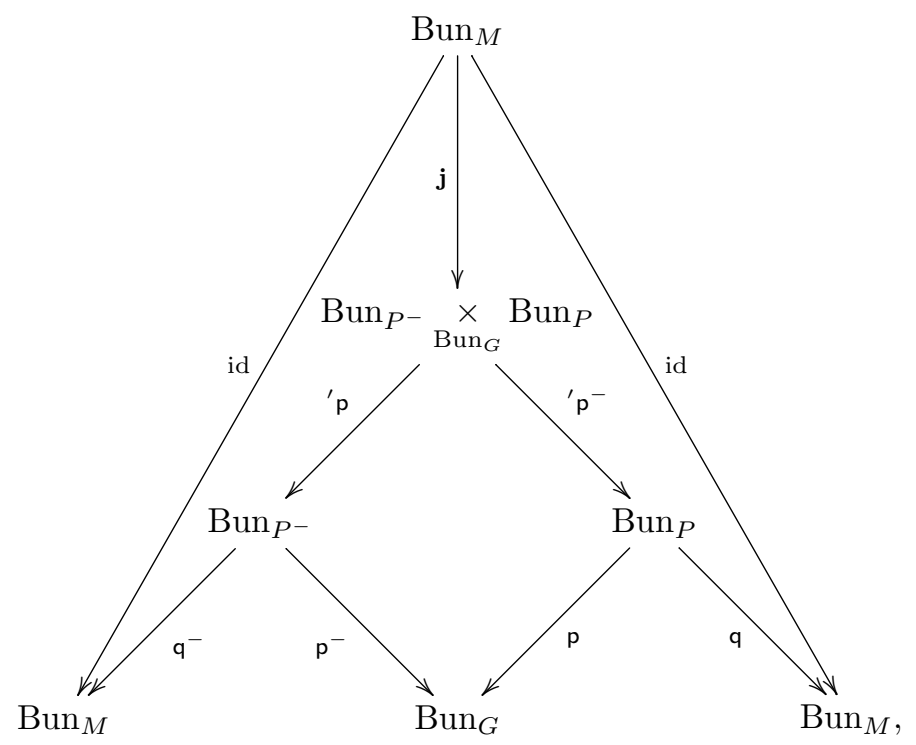

can be rewritten as

$$
(\mathrm{q}) ! \circ\left({ }^{\prime} \mathrm{p}^{-}\right) ! \circ\left({ }^{\prime} \mathrm{p}\right)^{*} \circ\left(\mathrm{q}^{-}\right)^{*} \text {. }
$$

The natural transformation

$$
\mathrm{Id}_{\mathrm{D}-\bmod \left(\operatorname{Bun}_{M}\right)} \rightarrow \mathrm{CT}_{!} \circ \mathrm{Eis}_{!}^{-}
$$

is given by

$$
\left(\operatorname{id}_{\mathrm{Bun}_{M}}\right) ! \circ\left(\operatorname{id}_{\mathrm{Bun}_{M}}\right)^{*}=(\mathrm{q}) ! \circ\left({ }^{\prime} \mathrm{p}^{-}\right) ! \circ \mathbf{j} ! \circ \mathbf{j}^{*} \circ\left({ }^{\prime} \mathrm{p}\right)^{*} \circ\left(\mathrm{q}^{-}\right)^{*} \rightarrow(\mathrm{q}) ! \circ\left({ }^{\prime} \mathrm{p}^{-}\right) ! \circ\left({ }^{\prime} \mathrm{p}\right)^{*} \circ\left(\mathrm{q}^{-}\right)^{*},
$$

where the second arrow comes from the $\left(\mathbf{j}_{!}, \mathbf{j}^{*}\right)$-adjunction.

The natural transformation

$$
\mathrm{Id}_{\mathrm{D}-\bmod \left(\operatorname{Bun}_{M}\right)} \rightarrow \mathrm{CT}_{!}^{-} \circ \text { Eis! }
$$

is described similarly, with the roles of $P$ and $P^{-}$swapped.

Base change along

$$
\begin{array}{ccc}
\operatorname{Bun}_{P^{-}} \underset{\operatorname{Bun}_{G}}{\times} \operatorname{Bun}_{P} & \longrightarrow \operatorname{Bun}_{P^{-}} \times \operatorname{Bun}_{P} \stackrel{\mathrm{q}^{-} \times \mathrm{q}}{\longrightarrow} \operatorname{Bun}_{M} \times \operatorname{Bun}_{M} \\
\downarrow & \mathrm{pu}^{-} \times \mathrm{p} \downarrow \\
\operatorname{Bun}_{G} \stackrel{\Delta_{\mathrm{Bun}_{G}}}{\longrightarrow} \operatorname{Bun}_{G} \times \operatorname{Bun}_{G}
\end{array}
$$

implies that the object

$$
\left(\mathrm{CT}_{!}^{-} \otimes \mathrm{CT}_{!}\right) \circ\left(\Delta_{\mathrm{Bun}_{G}}\right)_{!}\left(k_{\mathrm{Bun}_{G}}\right) \in \mathrm{D}-\bmod \left(\operatorname{Bun}_{M} \times \mathrm{Bun}_{M}\right)
$$

identifies with

$$
\left(\mathbf{q}^{-} \underset{\operatorname{Bun}_{G}}{\times} \mathbf{q}\right) !\left(k_{\operatorname{Bun}_{P^{-}}} \underset{\operatorname{Bun}_{G}}{\times} \operatorname{Bun}_{P}\right),
$$

where $\mathrm{q}^{-} \underset{\text { Bun }_{G}}{\times} \mathrm{q}$ denotes the map

$$
\operatorname{Bun}_{P^{-}} \underset{\operatorname{Bun}_{G}}{\times} \operatorname{Bun}_{P} \rightarrow \operatorname{Bun}_{P^{-}} \times \operatorname{Bun}_{P} \stackrel{q^{-} \times q}{\longrightarrow} \operatorname{Bun}_{M} \times \operatorname{Bun}_{M}
$$


Now, the above description of the unit of the adjunctions implies that both circuits in the diagram in Lemma 4.2.3 are equal to the map

$$
\left(\Delta_{\operatorname{Bun}_{M}}\right) !\left(k_{\mathrm{Bun}_{M}}\right) \rightarrow\left(\mathrm{q}^{-} \underset{\operatorname{Bun}_{G}}{\times} \mathbf{q}\right) !\left(k_{\mathrm{Bun}_{P-}} \underset{\operatorname{Bun}_{G}}{\times \operatorname{Bun}_{P}}\right),
$$

that corresponds to the open embedding

$$
\operatorname{Bun}_{M} \stackrel{\dot{j}}{\rightarrow} \operatorname{Bun}_{P^{-}} \underset{\text { Bun }_{G}}{\times} \operatorname{Bun}_{P}
$$

4.3. Proof of Theorem 3.1.5. We are finally ready to prove Theorem 3.1.5.

We proceed by induction on the semi-simple rank of $G$. The case of a torus follows immediately from Corollary 3.3.3. Hence, we will assume that the assertion holds for all proper Levi subgroups of $G$.

4.3.1. Theorem 4.1.2, together with the induction hypothesis, imply that the essential image of $\mathrm{D}-\bmod \left(\operatorname{Bun}_{G}\right)_{\mathrm{co}, \text { Eis }}$ under Ps-Id $\mathrm{Bun}_{G}$, generates D-mod(Bun $\left.{ }_{G}\right)_{\text {Eis }}$.

Corollary 3.3.3 implies that the essential image of $\mathrm{D}-\bmod \left(\operatorname{Bun}{ }_{G}\right)_{\mathrm{co}, \mathrm{cusp}}$ under $\mathrm{Ps}_{\mathrm{s}}-\mathrm{Id}_{\mathrm{Bun}_{G},}$ ! generates (in fact, equals) $\mathrm{D}-\bmod \left(\operatorname{Bun}_{G}\right)_{\text {cusp }}$.

Hence, it remains to show that Ps- $\operatorname{Id}_{\mathrm{Bun}_{G}, !}$ is fully faithful.

4.3.2. The fact that $\mathrm{Ps}_{\mathrm{S}}-\mathrm{Id}_{\mathrm{Bun}_{G}, !}$ induces an isomorphism

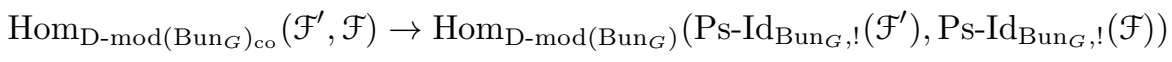

for $\mathcal{F}^{\prime} \in \mathrm{D}-\bmod \left(\operatorname{Bun}_{G}\right)_{\text {co,cusp }}$ follows from Proposition 3.3.5.

Hence, it remains to show that (4.6) is an isomorphism for $\mathcal{F}^{\prime} \in \mathrm{D}-\bmod \left(\operatorname{Bun}_{G}\right)_{\mathrm{co} \text {,Eis }}$. The latter amounts to showing that the functor $\mathrm{Ps}_{\mathrm{s}} \mathrm{Id}_{\mathrm{Bun}_{G}, !}$ induces an isomorphism

$$
\begin{aligned}
& \operatorname{Hom}_{\mathrm{D}-\bmod \left(\operatorname{Bun}_{G}\right)_{\mathrm{co}}}\left(\operatorname{Eis}_{\mathrm{co}, *}\left(\mathcal{F}_{M}\right), \mathcal{F}\right) \rightarrow
\end{aligned}
$$

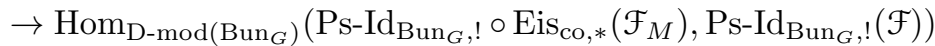

for $\mathcal{F}_{M} \in \mathrm{D}-\bmod \left(\operatorname{Bun}_{M}\right)_{\text {co }}$ for a proper parabolic $P$ with Levi quotient $M$.

4.3.3. Note that for $\mathcal{F}_{M} \in \mathrm{D}-\bmod \left(\operatorname{Bun}_{M}\right)_{\text {co }}$ and $\mathcal{F} \in \mathrm{D}-\bmod \left(\operatorname{Bun}_{G}\right)_{\text {co }}$ we have a commutative diagram:

$$
\begin{aligned}
& \operatorname{Hom}\left(\operatorname{Eis}_{\mathrm{co}, *}\left(\mathcal{F}_{M}\right), \mathcal{F}\right) \longrightarrow \operatorname{Hom}\left(\operatorname{Ps}-\operatorname{Id}_{\mathrm{Bun}_{G}, !} \circ \operatorname{Eis}_{\mathrm{co}, *}\left(\mathcal{F}_{M}\right), \operatorname{Ps}_{-I_{B_{n}, !},}(\mathcal{F})\right) \\
& \text { (4.3) } \downarrow \sim \\
& \left.\operatorname{Hom}\left(\text { Eis }_{!}^{-} \circ \operatorname{Ps}^{-I_{B_{1}}, !} \mathcal{F}_{M}\right), \mathrm{Ps}-\operatorname{Id}_{\mathrm{Bun}_{G}, !}(\mathcal{F})\right) \\
& \sim \\
& \sim \downarrow \\
& \operatorname{Hom}\left(\operatorname{Ps}_{-}-\operatorname{Id}_{\mathrm{Bun}_{M}, !}\left(\mathcal{F}_{M}\right), \mathrm{CT}_{*}^{-} \circ \mathrm{Ps}-\mathrm{Id}_{\mathrm{Bun}_{G}, !}(\mathcal{F})\right) \\
& \text { (4.4) } \uparrow \\
& \operatorname{Hom}\left(\mathcal{F}_{M}, \mathrm{CT}_{\mathrm{co}, ?}(\mathcal{F})\right) \longrightarrow \operatorname{Hom}\left(\operatorname{Ps}_{-I_{B_{n n}, !}}\left(\mathcal{F}_{M}\right), \operatorname{Ps}-\operatorname{Id}_{\mathrm{Bun}_{M}, !} \circ \mathrm{CT}_{\mathrm{co}, ?}(\mathcal{F})\right) .
\end{aligned}
$$

The bottom horizontal arrow in the above diagram is an isomorphism by the induction hypothesis. Now, Proposition 4.1.6 implies that the lower right vertical arrow is also an isomorphism. 
Hence, the upper horizontal arrow is also an isomorphism, as required.

\section{REFERENCES}

[AG] D. Arinkin and D. Gaitsgory, Singular support of coherent sheaves, and the geometric Langlands conjecture, arXiv:1201.6343.

[BG] A. Braverman, D. Gaitsgory, Geometric Eisenstein Series, Invent. Math 150 (2002), 287-384. Also: arXiv:math/9912097.

[BFGM] A. Braverman, M. Finkelberg, D. Gaitsgory and I. Mirkovic, Intersection cohomology of Drinfeld compactifications, Selecta Math (N.S.) 8 (2002), 381-418. Also: arXiv:math/0012129.

[DrGa1] V. Drinfeld and D. Gaitsgory, On some finiteness questions for algebraic stacks, Geometric and Functional Analysis 23 (2013), 149-294. Also: arXiv:1108.5351.

[DrGa2] V. Drinfeld and D. Gaitsgory, Compact generation of the category of D-modules on the stack of Gbundles on a curve, arXiv:1112.2402.

[DrGa3] V. Drinfeld and D. Gaitsgory, Geometric constant term functor(s), arXiv:1301.2071.

[Ga1] D. Gaitsgory, Outline of the proof of the geometric Langlands conjecture for $G L_{2}$, arXiv:1302.2506

[Ga2] D. Gaitsgory, Functors given by kernels, adjunctions, and duality, arXiv:1303.2763.

[Sch] S. Schieder, in preparation.

[Vi] E. B. Vinberg, On reductive algebraic semigroups, E. B. Dynkins Seminar, 145-182, Amer. Math. Soc. Transl. Ser. 2 169, Amer. Math. Soc., Providence, RI, 1995. 